\title{
PSD-Zip70 Deficiency Causes Prefrontal Hypofunction Associated with Glutamatergic Synapse Maturation Defects by Dysregulation of Rap2 Activity
}

\author{
Taira Mayanagi ${ }^{1}$, ${ }^{-H i r o k i}$ Yasuda ${ }^{2}$, and Kenji Sobue ${ }^{1}$ \\ ${ }^{1}$ Department of Neuroscience, Institute of Biomedical Sciences, School of Medicine, Iwate Medical University, 2-1-1 Yahaba, Shiwa 028-3694, Japan, and \\ ${ }^{2}$ Education and Research Support Center, Gunma University Graduate School of Medicine, 3-39-22 Showa-machi, Maebashi 371-8511, Japan
}

\begin{abstract}
Dysregulation of synapse formation and plasticity is closely related to the pathophysiology of psychiatric and neurodevelopmental disorders. The prefrontal cortex (PFC) is particularly important for executive functions such as working memory, cognition, and emotional control, which are impaired in the disorders. PSD-Zip70 (Lzts1/FEZ1) is a postsynaptic density (PSD) protein predominantly expressed in the frontal cortex, olfactory bulb, striatum, and hippocampus. Here we found that PSD-Zip70 knock-out (PSD-Zip70KO) mice exhibit working memory and cognitive defects, and enhanced anxiety-like behaviors. These abnormal behaviors are caused by impaired glutamatergic synapse transmission accompanied by tiny-headed immature dendritic spines in the PFC, due to aberrant Rap2 activation, which has roles in synapse formation and plasticity. PSD-Zip70 modulates the Rap2 activity by interacting with SPAR (spineassociated RapGAP) and PDZ-GEF1 (RapGEF) in the postsynapse. Furthermore, suppression of the aberrant Rap2 activation in the PFC rescued the behavioral defects in PSD-Zip70KO mice. Our data demonstrate a critical role for PSD-Zip70 in Rap2-dependent spine synapse development in the PFC and underscore the importance of this regulation in PFC-dependent behaviors.
\end{abstract}

Key words: glutamatergic synapse; maturation; prefrontal cortex; PSD-Zip70; Rap2; spine

Significance Statement

PSD-Zip70 deficiency causes behavioral defects in working memory and cognition, and enhanced anxiety due to prefrontal hypofunction. This study revealed that PSD-Zip70 plays essential roles in glutamatergic synapse maturation via modulation of the Rap2 activity in the PFC. PSD-Zip70 interacts with both SPAR (spine-associated RapGAP) and PDZ-GEF1 (RapGEF) and modulates the Rap2 activity in postsynaptic sites. Our results provide a novel Rap2-specific regulatory mechanism in synaptic maturation involving PSD-Zip70.

\section{Introduction}

The regulation of synapse formation and maturation is crucial for proper development of the CNS. Synaptic plasticity with the structural alteration of the spine is strongly associated with brain functions such as learning, memory, and cognition (Fu and $\mathrm{Zuo}$,

\footnotetext{
Received June 19, 2015; revised Aug. 27, 2015; accepted Sept. 13, 2015.

Author contributions: T.M. and K.S. designed research; T.M. and H.Y. performed research; T.M., H.Y., and K.S. analyzed data; T.M. and K.S. wrote the paper.

This work was supported by Japan Society for the Promotion of Science KAKENHI Grants 20240038, 23110510, and 25110728 to K.S., and 21700352 and 15 K21319 to T.M. We are grateful to Dr. Tsuyoshi Moira, Dr. Kentaro Fukumoto, and Dr. Daisuke Tanokashira for support with the breeding and backcrossings of PSD-Zip70K0 mice and beneficial comments. We are also grateful to the staff of the Center for In Vivo Sciences of Iwate Medical University for assistance for animal care.

The authors declare no competing financial interests.

Correspondence should be addressed to Kenji Sobue, Department of Neuroscience, Institute of Biomedical Sciences, School of Medicine, Iwate Medical University, 2-1-1 Yahaba, Shiwa 028-3694, Japan. E-mail: ksobue@iwate-med.ac.jp.

DOI:10.1523/JNEUROSCI.2349-15.2015

Copyright $\odot 2015$ the authors $\quad 0270-6474 / 15 / 3514327-14 \$ 15.00 / 0$
}

2011; Caroni et al., 2012; Sala and Segal, 2014; Nishiyama and Yasuda, 2015). It was recently demonstrated that glutamatergic synaptic dysfunction accompanied by morphological alteration of dendritic spines is closely related to the pathophysiology of psychiatric and neurodevelopmental disorders such as schizophrenia, depression, and autism spectrum disorder (ASD; Irwin et al., 2000; Hutsler and Zhang, 2010; Penzes et al., 2011; Bernardinelli et al., 2014). Recent genomic studies further revealed that a lot of synapse- and postsynaptic density (PSD)-related genes are candidate genetic risk factors for psychiatric and neurodevelopmental disorders (Bayés et al., 2011; Penzes et al., 2011; Chen et al., 2014; De Rubeis et al., 2014).

PSD-Zip70 is a PSD protein (Tachibana et al., 1999; Konno et al. 2002). A human ortholog of PSD-Zip70 is identical to a putative tumor suppressor, Lzts1/FEZ1 (Ishii et al., 1999; Vecchione et al., 2001). PSD-Zip70 is predominantly expressed in the brain, particularly in the frontal cortex, olfactory bulb, striatum, and hippocampus (Konno et al., 2002). PSD-Zip70 has been pro- 
a
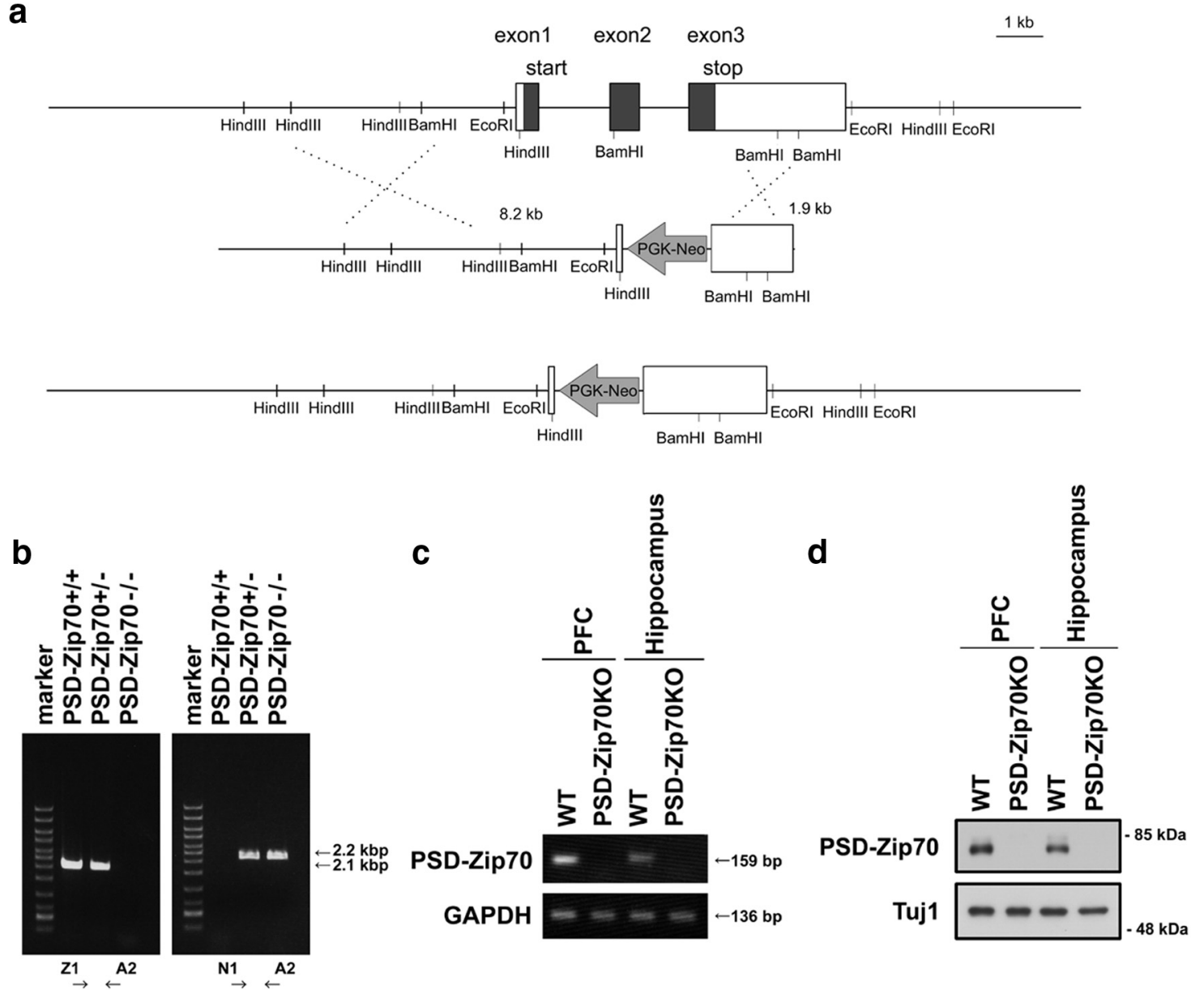

Figure 1. Generation of PSD-Zip70 (Lzts1)-targeted knock-out mice. $\boldsymbol{a}$, Schematics, from top to bottom, of the WT allele, targeting construct, and K0 allele. The schematic of the WT Lzts 19 genomic allele shows exons $1-3$ and important restriction enzyme recognition sites. The $K 0$ allele is the Lzts 1 locus after homologous recombination with the targeting construct. The location of the PGK-Neo cassette is shown. The primers used for PCR genotyping are indicated on all relevant alleles. $\boldsymbol{b}, \mathrm{PCR}$-based genotyping of the WT allele ( $2.1 \mathrm{kbp})$ compared with the K0 allele ( $2.2 \mathrm{kbp})$ using primers $\mathrm{Z1}$ and A2, N1 and A2, respectively. c, Representative semiquantitative reverse transcriptase-PCR results for PSD-Zip70 in the PFC and hippocampus from WT and PSD-Zip70KO (hom0zygous) mice. GAPDH was used as an internal control. $\boldsymbol{d}$, Western blot analysis of SDS lysates of the PFC and hippocampus from 8-week-old WT and PSD-Zip70KO homozygous mice were analyzed using an anti-PSD-Zip70 antibody with Tuj1 as the loading control.

posed to function in spine formation and maturation in cooperation with its interacting partner, SPAR (Maruoka et al., 2005). SPAR is a spine-associated RapGAP (Pak et al., 2001). Rap1 and Rap2, members of the Rap family of small GTPases, play important roles in synaptic remodeling and plasticity (J.J. Zhu et al., 2002; Y. Zhu et al., 2005; Fu et al., 2007).

Here we generated PSD-Zip70 knock-out (PSD-Zip70KO) mice, which exhibit impaired working memory, cognition, and enhanced anxiety-like behaviors, defects that are associated with psychiatric and neurodevelopmental disorders. The prefrontal cortex (PFC), in which PSD-Zip70 is highly expressed, is critically important for certain aspects of behavior. We demonstrated that the PSD-Zip70 deficiency causes PFC dysfunction due to defects in glutamatergic synaptic transmission and impaired spine maturation through aberrant activation of Rap2.

\section{Materials and Methods}

Generation of PSD-Zip70-knock-out mice. The strategy for creating knock-out mice of the PSD-Zip70 (Lzts1) gene is diagrammed in Figure $1 a$. Fragments representing $8.2 \mathrm{~kb}$ of the upstream flanking region of exon 1 and $1.9 \mathrm{~kb}$ of the downstream flanking region of exon 3 were retrieved from the BAC library derived from the $\mathrm{C} 57 \mathrm{BL} / 6 \mathrm{~J}$ mouse genome. The PGK-Neo cassette was inserted in the reverse direction of the Lzts 1 gene. The resulting plasmid was linearized and electroporated into the mouse embryonic stem (ES) cell line, iTL IC1, derived from C57BL/ 6 NTac. A selected positive clone was expanded and injected into blastocysts of a BALB/c mouse. ES cells were incorporated into the germ line and produced fertile, chimeric males.

For genotyping, PCR was performed on tail samples using the wild-type (WT) allele- and knock-out (KO) allele-specific primer sets. A forward primer specific for the WT allele (primer Z1, 5'-GAGGACATCATAGC CACTGAGA- $3^{\prime}$ ), a forward primer that annealed to the Neo cassette (primer N1, 5'-TGCGAGGCCAGAGGCCAGTTGTGTAGC-3'), and a common reverse primer that annealed to the downstream region of the Lzts 1 gene (primer A2, 5'-AGCAGACTTGGGAAGGTATCTG-3') were used. Primers $\mathrm{Z} 1$ and $\mathrm{A} 2$ detected the WT allele (2.1 kbp), and primers N1 and A2 detected $\mathrm{KO}$ allele (2.2 kbp), respectively (Fig. 1b). The PSD-Zip70KO mice were backcrossed to $\mathrm{C} 57 \mathrm{BL} / 6 \mathrm{~J}$ for $>10$ generations. The homozygous PSDZip70KO mice were viable, reached maturity, and were fertile. The PSDZip70KO mice developed several tumors, although the onset was quite late (18 months), similar to those in a previous report (Vecchione et al., 2007). In the present study, the data were obtained before the onset of tumors in the PSD-Zip70KO mice.

Animals and behavioral tests. Animals were housed in groups with a $12 \mathrm{~h}$ light/dark cycle (lights on 7:00 A.M.-7:00 P.M.) in the Center for In Vivo Sciences of Iwate Medical University, the Center for the Institute of Experimental Animal Science of Osaka University, and the Institute of Experimental Animal Research, Gunma University Graduate School of Medicine. All of the procedures involving animals and their care were 
approved by the Animal Care Committees of these institutes, and were performed according to their guidelines for animal experiments. For the behavioral studies, 8- to 10-week-old male mice were tested at 5:00-7:30 P.M. The mice were allowed to habituate to the room for $0.5-1 \mathrm{~h}$ before testing. Mouse order was randomized. Behavior was observed and recorded under light with a camera mounted above the field for $5 \mathrm{~min}$. The behavior and the parameters were analyzed using automated tracing software, ANY-maze (Stoelting Co.).

The open-field apparatus consisted of a gray plastic chamber $(60 \times$ $60 \times 15 \mathrm{~cm}$ ) with a white floor. A mouse was placed in the center of the field. The following behavioral variables were analyzed using the automated tracing software ANY-maze: the track traveled by the mouse, traveled distance, and time spent in the center zone (the central $30 \times 30 \mathrm{~cm}$ section of the open field).

The Y-maze consisted of three equally spaced arms $(30 \mathrm{~cm}$ long, $5 \mathrm{~cm}$ wide, and $7 \mathrm{~cm}$ high). A mouse was released at the distal end of one arm. The sequence of arm entries and total number of arm visits were analyzed using ANY-maze. The total number of arm entries is considered to reflect the locomotor activity. An alternation was defined as three successive entries into the three separate arms. The alternation score was the number of alternations divided by the number of total entries minus two.

The elevated plus maze consisted of four arms $30 \mathrm{~cm}$ long and $5 \mathrm{~cm}$ wide, extending from a central platform $\left(5 \mathrm{~cm}^{2}\right)$ and placed on an elevated base ( $50 \mathrm{~cm}$ high). Two opposite arms were enclosed by a wall (15 $\mathrm{cm}$ high), and the other two arms were open. A mouse was placed on the central platform.

The novel object recognition test was performed the day after the open field test, which served as the habituation (no object). Twenty-four hours after habituation, the mouse was exposed to the familiar field with two identical objects (dark-pink cylinders) placed at an equal distance (familiarization, training trial). After another $24 \mathrm{~h}$, the mouse was allowed to explore the open field in the presence of the familiar object and a novel object (light-green cone), which were approximately consistent in height and volume, but different in shape and appearance (test trial). The time spent exploring each object was analyzed.

The social interaction test was performed using an apparatus consisting of three chambers dividing clear walls $(20 \times 45 \times 15 \mathrm{~cm}$, each chamber), which allows free access to each chamber. Two identical containers were placed at the center of each side chamber. One contained the unfamiliar mouse (social) and the other contained a green cone (object). The time spent within each chamber was analyzed.

Primary neuron culture. Cultured neurons were prepared from mouse embryonic brain on embryonic day 16.5 (E16.5). The frontal cortices, mainly parts of the medial PFC, were dispersed with $0.02 \%$ trypsin in HBSS (Sigma), and the cell suspension was plated onto $1.0 \mathrm{mg} / \mathrm{ml}$ polyL-lysine-coated glass coverslips at $10,000-15,000 \mathrm{cells} / \mathrm{cm}^{2}$. The neurons were cultured in glial-conditioned minimum essential medium (MEM) containing $1 \mathrm{~mm}$ sodium pyruvate, $0.6 \%(\mathrm{w} / \mathrm{v}) \mathrm{D}$-glucose, and 2\% B27 supplement (Life Technologies) in a $37^{\circ} \mathrm{C}$ incubator with $5 \% \mathrm{CO}_{2}$. After $3 \mathrm{~d}$, an equal volume of fresh glial-conditioned medium was added.

Expression plasmids and transfection. The coding regions of mouse PSDZip70, Rap2, and rat SPAR were amplified by PCR and subcloned into the highly efficient mammalian expression plasmid pCAGGS. Plasmidcontaining human PDZ-GEF1, and PDZ-GEF2 were obtained from Promega. A Myc-, FLAG-, or HA-tag sequence was added to the $3^{\prime}$ - (PSDZip70) or 5' (Rap2, SPAR, PDZ-GEF1, and PDZ-GEF2)-end of the coding sequence. A GAP-inactive form of SPAR (SPAR-GAPmut; Pak et al., 2001), CA-Rap2 (Rap2V12), and DN-Rap2 (Rap2N17; Ryu et al., 2008) were constructed as previously described. For miRNA-mediated knockdown, plasmids expressing miRNA were constructed using the Block-iT Pol II miR RNAi Expression Vector kit (Life Technologies). The target sequences were as follows: PSD-Zip70 miRNA: 5'-AAGGCTCTGTCGTTCTCTGAT-3', Rap2a miRNA: 5'-GCTGTTCTGCCTGTAACATAC-3', Rap2b miRNA: 5'-GTGAACGTGAGGTCTCCTATG-3', Rap2c miRNA: 5' -CAAGATCA GTGTTGTACAACT-3', PDZ-GEF1 miRNA: 5'-GAAGAGCTGGATTCC TGGTCA-3', PDZ-GEF2 miRNA: 5'-TGAGGAGAAGTTCCAGATGAT$3^{\prime}$. The coding regions of EmGFP and the miRNAs were amplified from target sequence-inserted pcDNA6.2-GW/EmGFP-miR plasmids by PCR and subcloned into pCAGGS. Each knockdown efficacy was validated by cotransfection with the expression plasmids in HEK293T cells (data not shown).

Cultured neurons were transfected by the calcium phosphate method as described previously (Jiang and Chen, 2006). Neurons were transfected at $0-1$ day in vitro (DIV). For experiments using heterologous HEK293T cells, the cells were transfected with expression vectors using Lipofectamine 2000 (Life Technologies). In utero electroporation was performed at E14.5, as described previously (Fukumoto et al., 2009; Niwa et al., 2010). In brief, pregnant mice were deeply anesthetized, and 1-2 $\mu \mathrm{l}$ of plasmid solution including $0.25 \mathrm{mg} / \mathrm{ml}$ Fast Green (Sigma) was administered into the intraventricular region of the embryonic brain, followed by electroporation. Electric pulses were generated by a CUY21 EDIT electroporator (BEX) and applied to the cerebral wall as five repeats of $35 \mathrm{~V}$ for $50 \mathrm{~ms}$, with an interval of $950 \mathrm{~ms}$. All of the expression plasmids were used at a concentration of $2 \mathrm{mg} / \mathrm{ml}$.

Imaging. Procedures for immunocytochemistry and immunohistochemistry were essentially performed as described previously (Fukumoto et al., 2009). Golgi staining was performed using a modified Golgi-Cox impregnation method. The brains of 10 -week-old male littermates were processed with an FD Rapid Golgi stain kit (FD NeuroTechnologies) following the manufacturer's protocol. Stained samples were observed under a BIOREVO BZ-9000 (Keyence) fluorescence microscope with a $60 \times$ [numerical aperture (NA) 1.4] oil-immersion lens or under an Axiovert $200 \mathrm{M}$ fluorescence microscope (Carl Zeiss) with a $60 \times$ (NA 1.4) oil-immersion lens. Fluorescent images were contrast-enhanced using Adobe Photoshop software, and fluorescence intensity was quantified by ImageJ software. A heat map for signal intensity was generated by the HeatMap Histogram plug-in for the ImageJ software.

Antibodies. The anti-PSD-Zip70 (C terminus; Konno et al., 2002), anti-SPAR, anti-bassoon (Maruoka et al., 2005), anti-synapsin I (Okabe and Sobue, 1987), and anti-GKAP (Usui et al., 2003) antibodies were described previously. The following antibodies were purchased: antiFLAG (F1804, F7425), anti-transferrin receptor (TfR; HPA028598), anti-GAD65/67 (G5163), and anti- $\alpha$-tubulin (T9026) from Sigma; antic- $M y c$ (sc-40, sc-789), anti-RhoA (sc-418), anti-Rap1 (sc-65), anti-SPAR (sc-20846), anti-Cdc42 (Sc-87), anti-GAPDH (sc-25778), anti-p38 (sc535), anti-JNK1 (sc-474), anti-phospho-ERK1/2 (sc-7383), anti-ERK1 (sc-93-G), anti-ERK2 (sc-154-G), anti-Myc (sc-789) antibodies, and horseradish peroxidase (HRP)-conjugated anti-goat IgG (sc-2020) from Santa Cruz Biotechnology; anti-Ras (R02120), anti-Rap2 (610216), antiNR1 (556308), and anti-FEZ1 (611710) from BD Transduction Laboratories; anti-vGlut1 (MAB5502), anti-GluR2 (extracellular; MAB397), anti-pSer880-GluR2 (MABN103), anti-NR2B (AB1557P), anti-Rac1 (23A8), anti-GluR1 (06-306), and anti-GluR2/3 (AB1506) from Millipore; anti-GluR4 (catalog \#8040), anti-pSer845-GluR1 (catalog \#8084), anti-pTyr(869/873/876)-GluR2 (catalog \#3921), anti-phospho-p38 (catalog \#9211), and anti-phospho-JNK (catalog \#9255) antibodies from Cell Signaling Technology; anti-PSD-95 (MA1-045) from Affinity BioReagents; anti-NR2A (3916-1) from Epitomics; anti-HA (3F10) from Roche; anti-Tuj1 (MMS-435P) from Covance; anti-PDZ-GEF1 (H00009693) from Abnova; anti-Shank3 (NBP1-47610) from Novus; anti-PDZ-GEF2 (A301-967) from Bethyl; anti-Halo-tag (G9281) from Promega; anti-VGAT (131-002) antibodies from SYnaptic SYstems; and HRP-conjugated anti-rabbit IgG (NA934) and HRP-conjugated antimouse IgG (NA931) from GE Healthcare. The anti-GFP (A-11120, A-11122) and all of the Alexa Fluor-conjugated secondary antibodies were purchased from Life Technologies.

Protein analyses. For co-immunoprecipitation (co-IP) assays using heterologous cells, HEK293T cells transfected with expression vectors were lysed with lysis buffer [ $1 \%$ Triton X-100, pH 7.5, $0.2 \%$ sodium deoxycholate (DOC), 20 mм Tris- $\mathrm{HCl}, 150 \mathrm{~mm} \mathrm{NaCl}, 1 \%$ Protease Inhibitor Cocktail (Nacalai), and 1\% Phosphatase Inhibitor Cocktail (Nacalai)]). For co-IP assays using brain samples, the frontal cortices were collected from 10-week-old male mice. The cortices were lysed with DOC-lysis buffer [1\% Triton X-100, pH 7.5, 1\% DOC, 20 mм Tris-HCl, $150 \mathrm{~mm} \mathrm{NaCl}, 1 \%$ Protease Inhibitor Cocktail, and 1\% Phosphatase Inhibitor Cocktail] with sonication, and then the lysate was diluted with lysis buffer without DOC to a final DOC concentration of $0.2 \%$. After preclearing the sample with Sepharose $4 \mathrm{~B}$ beads, co-IP was performed 
using the indicated antibodies and protein G-Sepharose (GE Healthcare). The Sepharose beads were boiled in SDS-sample buffer to elute the immunocomplexes.

The assay for GTP-bound small GTPase levels was based on the Rap1 Activation Assay Kit (Millipore). Cultured neurons or individual frontal cortices from mice were used. Pull-down assays were then performed using RalGDS-RBD beads for the Rap1, Rap2, and Ras activities, Rhotekin-RBD beads (Millipore) for the Rho activity, and PAK-PBD beads (Millipore) for the Cdc42 and Racl activities. As a positive control, GTP $\gamma \mathrm{S}$ (Sigma) was used. The proteins eluted from the beads were analyzed by Western blots.

For surface biotinylation assay, cortical slices (400 $\mu \mathrm{m}$ thick) were prepared from 8 - to 10 -week-old male mice and the prefrontal cortices were collected. The surface proteins were labeled with $0.3 \mathrm{mg} / \mathrm{ml}$ SulfoNHS-SS-biotin (Pierce) in HBSS for 15 min at $4^{\circ} \mathrm{C}$. After incubation, the unbound biotin was quenched by Tris buffer ( $50 \mathrm{~mm}$ Tris- $\mathrm{HCl}, \mathrm{pH} 8.0$, $120 \mathrm{~mm} \mathrm{NaCl}$ ). The tissues were then lysed with $1 \%$ Triton X-100, $0.1 \%$ SDS, $20 \mathrm{~mm}$ Tris- $\mathrm{HCl}, \mathrm{pH}$ 7.5, $150 \mathrm{~mm} \mathrm{NaCl}, 1 \%$ Protease Inhibitor Cocktail, and 1\% Phosphatase Inhibitor Cocktail. The biotinylated proteins were isolated by Streptavidin-Agarose (Life Technologies) and analyzed by Western blots for each protein.

The procedure for Western analysis was essentially performed as described previously (Fukumoto et al., 2009). The detected band intensities were quantified using ImageJ software.

Slice electrophysiology. Coronal slices were prepared from postnatal day 59-88 PSD-Zip70KO mice or their WT littermates by using the protective recovery method (Zhao et al., 2011; Tomioka et al., 2014). Mice were anesthetized with isoflurane and sodium pentobarbital and transcardially perfused with a room-temperature carbogenated $\left(95 \% \mathrm{O}_{2}\right.$ and $5 \% \mathrm{CO}_{2}$ ) protective artificial CSF (ACSF) containing (in mм) $92 \mathrm{~N}$-methyl-D-glucamine (NMDG), $2.5 \mathrm{KCl}, 1.2 \mathrm{NaH}_{2} \mathrm{PO}_{4}, 30$ $\mathrm{NaHCO}_{3}, 20$ HEPES, 25 glucose, 5 ascorbate, 3 sodium pyruvate, 12 $\mathrm{N}$-acetyl-L-cysteine, $0.5 \mathrm{CaCl}_{2}$, and $10 \mathrm{MgSO}_{4}$. The mice were then decapitated, the brains were removed, and coronal slices were cut using a vibrating blade microtome (VT1200S, Leica) at room temperature. Slices were recovered in the carbogenated protective ACSF at $32^{\circ} \mathrm{C}$ for $12 \mathrm{~min}$ and then transferred into a holding chamber containing a roomtemperature carbogenated incubating ACSF of the following composition (in mu): $119 \mathrm{NaCl}, 2.5 \mathrm{KCl}, 1 \mathrm{NaH}_{2} \mathrm{PO}_{4}, 26.2 \mathrm{NaHCO}_{3}, 12.5$ glucose, $2 \mathrm{CaCl}_{2} \cdot 4 \mathrm{H}_{2} \mathrm{O}, 2 \mathrm{MgSO}_{4} \cdot 7 \mathrm{H}_{2} \mathrm{O}, 5 \mathrm{HEPES}$, and $12 \mathrm{~N}$-acetyl-Lcysteine. After recovery for at least $2 \mathrm{~h}$, the slices were transferred to a recording chamber mounted on an upright microscope (BX51WI, Olympus) equipped with IR-DIC optics. Slices were perfused with a carbogenated recording ACSF (at $\sim 32^{\circ} \mathrm{C}$ ) containing (in mM) $119 \mathrm{NaCl}$, $2.5 \mathrm{KCl}, 4.0 \mathrm{CaCl}_{2}, 4.0 \mathrm{MgSO}_{4}, 1.0 \mathrm{NaH}_{2} \mathrm{PO}_{4}, 26.2 \mathrm{NaHCO}_{3}, 11$ glucose, 5 HEPES, and 0.1 picrotoxin. When measuring AMPAR/NMDAR ratios in synapses on layer $2 / 3$ pyramidal cells of the medial prefrontal cortex including the prelimbic, infralimbic, and anterior cingulate cortex, afferents to these cells were activated by a patch pipette that had a broken tip, filled with recording ACSF, and placed $\sim 80-100 \mu \mathrm{m}$ beneath the cell. These pyramidal cells were patched and EPSCs were recorded using a MultiClamp 700A (Molecular Devices) through a glass electrode filled with an internal solution containing (in mM) 135 cesium methanesulfonate, 10 HEPES, 0.2 EGTA, $8 \mathrm{NaCl}, 4 \mathrm{Mg}$-ATP, $0.3 \mathrm{Na}_{3} \mathrm{GTP}$ ( $\mathrm{pH} 7.2$ with $\mathrm{CsOH}$, osmolarity adjusted to $285 \mathrm{mOsm}$ ). The AMPAR/NMDAR ratio was calculated as the ratio of the amplitude of EPSCs at $-70 \mathrm{mV}$ to that of EPSCs at $+40 \mathrm{mV}$. Decays of averaged EPSCs at $+40 \mathrm{mV}$ were calculated as reported previously (Yasuda and Mukai, 2015). Miniature EPSCs (mEPSCs) were recorded in the presence of $1 \mu \mathrm{M}$ TTX. Data acquisition and analysis were performed using custom Igor Pro (WaveMetrics) software routines.

Semiquantitative reverse transcription-PCR. The total RNA was extracted from brain tissues using TRIzol reagent (Life Technologies) and reverse-transcribed with SuperScript VILO Master Mix (Life Technologies). The cDNA was amplified with gene-specific primer pairs using SYBR GreenER qPCR SuperMix Universal reagent (Life Technologies). The primer sequences used in this study are as follows: PSD-Zip70-F: 5'-CCATCGCCCAGACTACACAG-3', PSD-Zip70-R: 5' -ACAGGCTT GAAGGCAGTGG-3', GAPDH-F: 5' -CGTGCCGCCTGGAGAAAC-3', GAPDH-R: 5'-TGGGAGTTGCTGTTGAAGTCG-3'.
Statistical analysis. All values represent means \pm SEM. All transfection experiments were performed at least three times. The described " $n$ " indicates biological replicates. We used Excel Statistics add-in software (Social Survey Research Information Co., Ltd) for statistical processing. Statistical differences between pairs of values were analyzed by Student's $t$ test. One-way ANOVA with Tukey-Kramer post hoc test was used for group comparisons. Fisher's protected least significant difference test as a post hoc test was used to analyze the data (see Fig. $8 b$ ). $p<0.05$ was considered significant.

\section{Results}

\section{Generation of PSD-Zip70KO mice}

To evaluate the neural functions of PSD-Zip70 in vivo, we newly generated PSD-Zip70-deficient mice (Fig. 1a,b). A human ortholog of PSD-Zip70 is identical to Lzts1, which has been reported to have a role in carcinogenesis (Ishii et al., 1999; Vecchione et al., 2001). Lzts1 KO mice develop various tumors with late onset (Vecchione et al., 2007), but no neuronal analysis of these mice has been performed. In a previous report, $\mathrm{KO}$ mice were produced by introducing a stop codon 123 bp downstream of the first start (ATG) codon. Our strategy for targeted $\mathrm{KO}$ of the PSD-Zip70 (Lzts1) gene was to delete all of the coding regions of the three exons starting upstream of the ORF, because the $\mathrm{N}$ terminus of PSD-Zip70 possesses a myristoylation site that is important for the protein's membrane targeting (Konno et al., 2002). Homozygotes of PSD-Zip70KO mice were born in the expected Mendelian ratio, and showed no differences from WT mice in their growth, survival, or fertility for at least 12 months after birth (data not shown). The expression of PSD-Zip70 mRNA and protein was completely lacking in PSD-Zip70KO mice (Fig. $1 c, d$ ).

\section{Working memory and cognition defects and enhanced anxiety in the PSD-Zip70KO mice}

We examined the behavioral properties of the PSD-Zip70KO mice, particularly those related to the PFC and hippocampus, because PSD-Zip70 is highly expressed in these regions (Konno et al., 2002). First, in the open-field test, PSD-Zip70KO mice displayed normal spontaneous locomotor activity but a significant tendency to avoid entry into the center zone (Fig. 2a), which indicates increased anxiety. We then assessed the anxiety response using an elevated plus maze test. The PSD-Zip70KO mice spent a shorter time in the open arm of the maze (Fig. $2 b$ ). Thus, the PSD-Zip70KO mice exhibited enhanced anxiety-related behaviors.

In the Y-maze test, PSD-Zip70KO mice displayed normal locomotor activity, but their alternation score was significantly decreased (Fig. 2c). This decreased spatial novelty preference in the Y-maze test is regarded as impaired spatial working memory. Next, a novel object recognition test was used to evaluate cognition, particularly recognition memory. The PSD-Zip70KO mice showed a lower preference to access a novel object. The result indicates impaired cognition (Fig. $2 d$ ). We also evaluated sociability by the three-chamber social interaction test (Fig. 2e). There were no significant differences in sociability in the PSD-Zip70KO mice.

\section{Defect in AMPAR-mediated glutamatergic synaptic transmission and morphological abnormalities in dendritic spines in PSD-Zip70KO neurons}

The enhanced anxiety, and impaired working memory and cognition of the PSD-Zip70KO mice suggested altered activity of the medial prefrontal cortex (mPFC), which is known to be important for emotional and cognitive aspects of behavior (Levy and Goldman-Rakic, 2000; Ragland et al., 2007; Euston et al., 2012). To investigate the functional alteration of synaptic transmission 
a

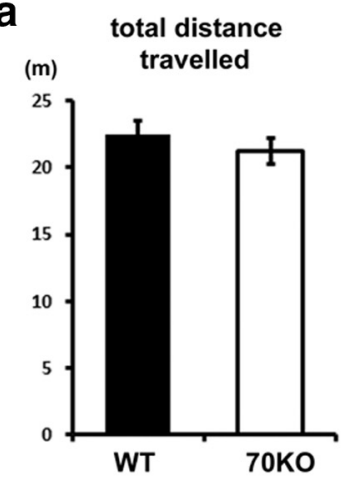

C
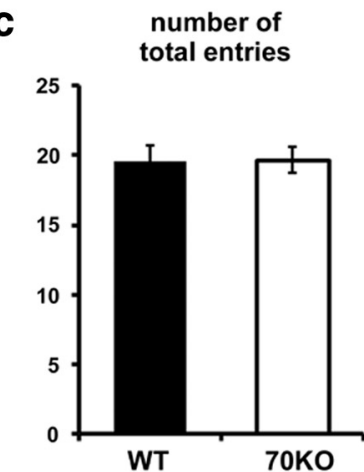

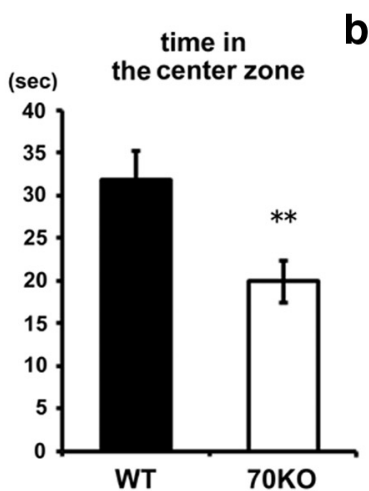

WT
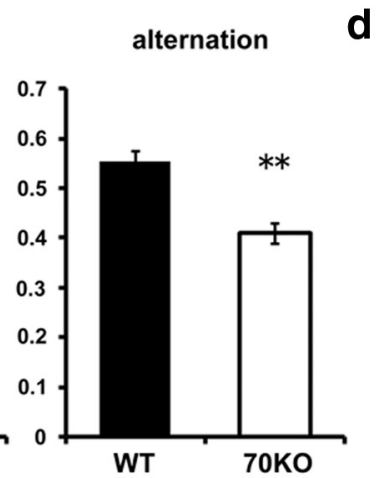

b time in (sec) the open zone

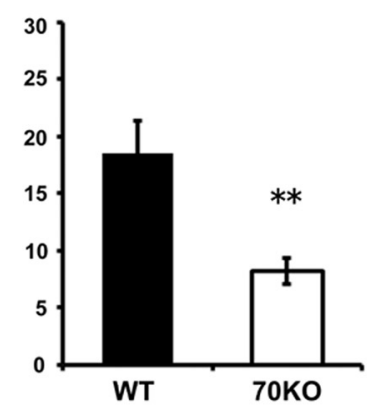

time in

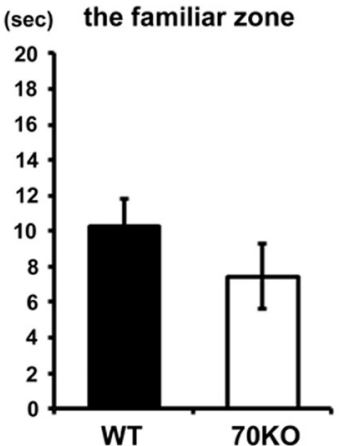

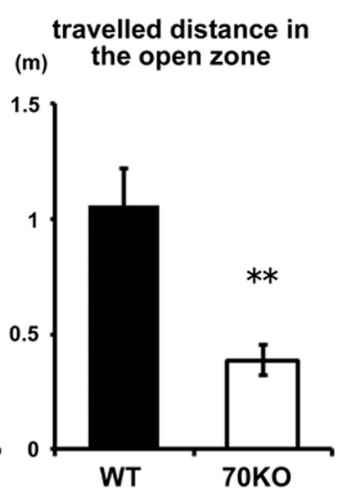

time in

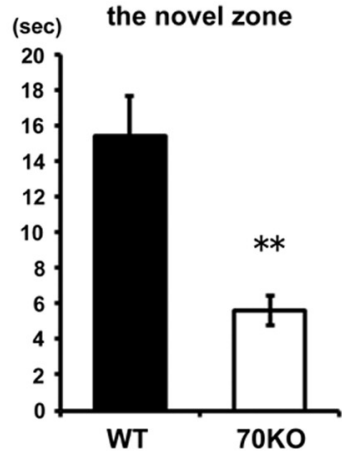

e
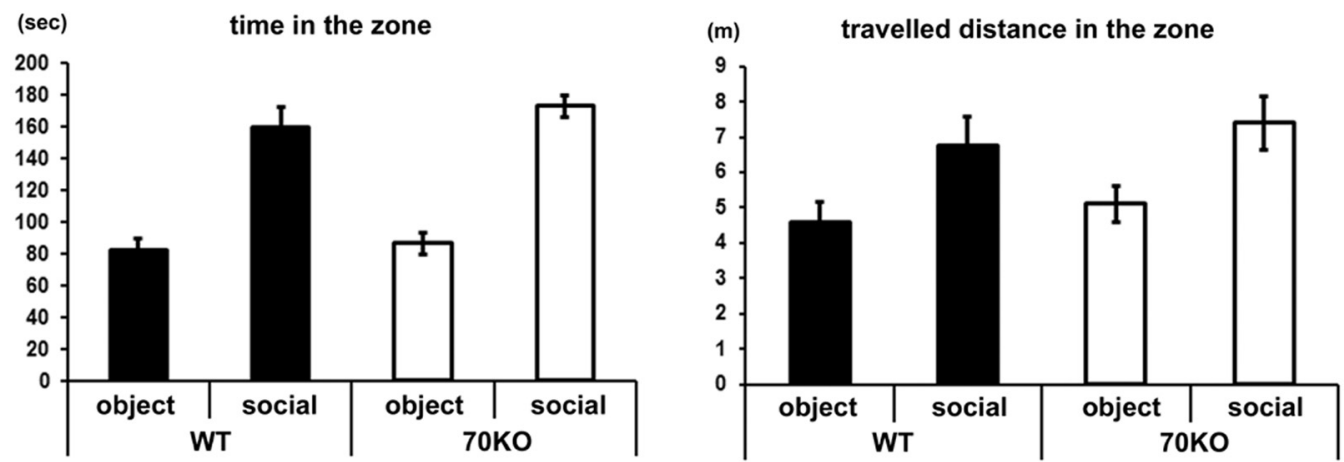

Figure 2. Behavioral properties of PSD-Zip70KO mice. $\boldsymbol{a}$, Open-field test. Although the locomotor activity (track length) was similar in WT and PSD-Zip70KO (70K0) mice (left), PSD-Zip70K0 mice showed a significant tendency to avoid entry into the center zone (right; $t=3.42, p=0.001 ; \mathrm{WT}, n=21 ; \mathrm{PSD}$-Zip70K0, $n=23$ ). $\boldsymbol{b}$, Elevated plus maze test. PSD-Zip70K0 mice had a significant tendency to avoid entering open arms. PSD-Zip70K0 mice also exhibited shorter durations (left; $t=3.52, p<0.001$ ) and track lengths (right; $t=4.11, p<0.001$ ) in the open arms (open zone; WT, $n=33$; PSD-Zip70KO, $n=38$ ). c, Y-maze test. PSD-Zip70K0 mice displayed normal locomotor activity (left), but their alternation score was significantly decreased (right; $t=5.23, p<0.001$; WT, $n=24$; PSD-Zip70KO, $n=29$ ). $\boldsymbol{d}$, Novel object recognition test. A training trial was performed using the same two objects, and a test trial was performed $24 \mathrm{~h}$ later. In the test trial, the time spent in the zones $(20 \mathrm{~cm} \times 20 \mathrm{~cm}$ each) around the familiar object (familiar zone) or the novel object (novel zone) was recorded. PSD-Zip70K0 mice exhibited lower preference to access the novel object (right; $t=4.25, p<0.001 ; \mathrm{WT}, n=19 ;$ PSD-Zip70K0, $n=18$ ). $\boldsymbol{e}$, Three-chamber social interaction test. There were no significant differences in sociability between the PSD-Zip70K0 and WT mice (WT, $n=11$; PSD-Zip70K0, $n=11)$. Error bars indicate SEM. Student's $t$ test. ${ }^{*} p<0.05,{ }^{* *} p<0.01$.

caused by PSD-Zip70 deficiency, we performed electrophysiological recording from pyramidal cells in layer $2 / 3$ of the mPFC. Although the mean mEPSC amplitude was not altered (WT, $6.48 \pm 0.30 \mathrm{pA}$; PSD-Zip70KO, $6.32 \pm 0.25 \mathrm{pA}$ ), the mEPSC frequency was markedly reduced, $>50 \%$, in PSD-Zip70KO neurons compared with WT neurons (WT, $7.49 \pm 1.30 \mathrm{~Hz}$; PSDZip70KO, $3.47 \pm 0.55 \mathrm{~Hz}$; Fig. 3a,b).

Because PSD-Zip70 is predominantly localized to postsynaptic sites and absent from presynaptic sites including axons (Konno et al., 2002), we examined the AMPAR/NMDAR ratio to evaluate the postsynaptic properties. We found that the AMPAR/ NMDAR ratio was decreased in the PSD-Zip70KO neurons (WT, $1.74 \pm 0.3$; PSD-Zip70KO, $1.15 \pm 0.09$ ), which suggests impaired AMPAR-mediated synaptic transmission (Fig. $3 c$,d). In addition, decays of EPSCs at $+40 \mathrm{mV}$ were prolonged in PSD-Zip70KO neurons (WT, $94.1 \pm 8.4 \mathrm{~ms}$; PSD-Zip70KO, $124.6 \pm 12.2 \mathrm{~ms}$; Fig. 3e). The data suggest that NR2B-containing immature-type NMDARs are possibly dominant in the postsynaptic sites of PSDZip70KO neurons. Notably, NR2B-containing NMDARs are reported to be dominant in immature postsynapses such as "silent synapses," which express NMDARs but not AMPARs, at the postsynaptic surface (Isaac et al., 1995; Rumpel et al., 1998).

The state of synaptic development and the efficiency of synaptic transmission are both correlated with the morphological features of dendritic spines (Matsuzaki et al., 2001; Nimchinsky et al. 2002; Caroni et al., 2012). Golgi staining for pyramidal neurons in layer $2 / 3$ of the mPFC revealed that the frequency of thin-headed spines in both the apical and basal dendrites was 
a

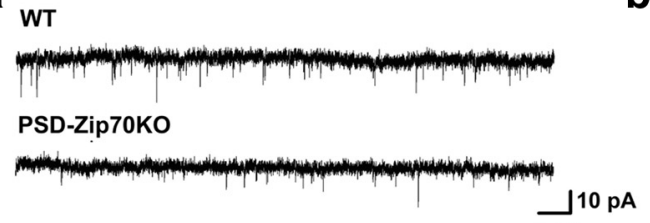

C

WT

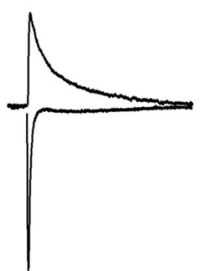

PSD-Zip70KO

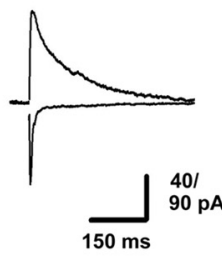

b

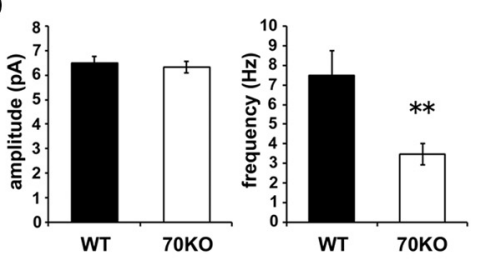

d

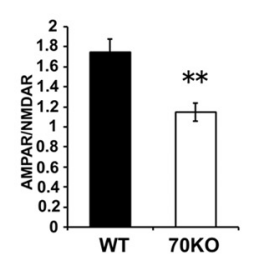

e

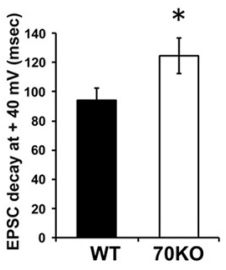

f

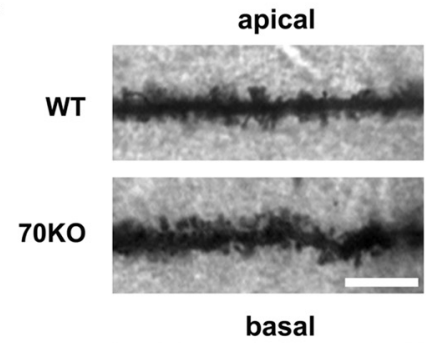

WT

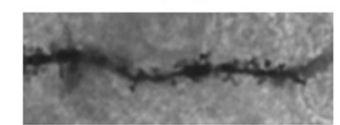

$70 \mathrm{KO}$

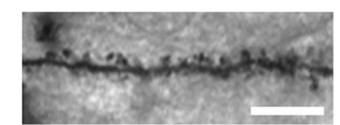

$\mathbf{h}$

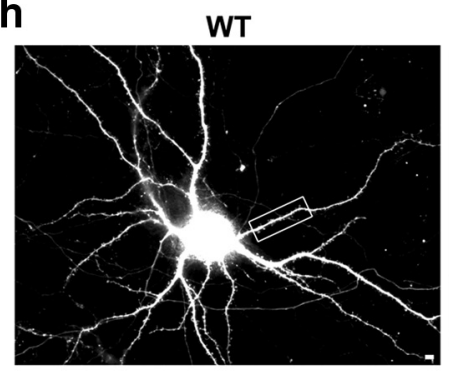

PSD-Zip70KO

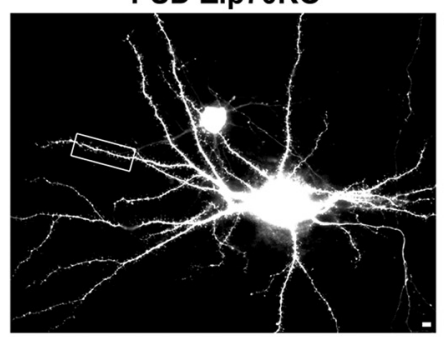

g
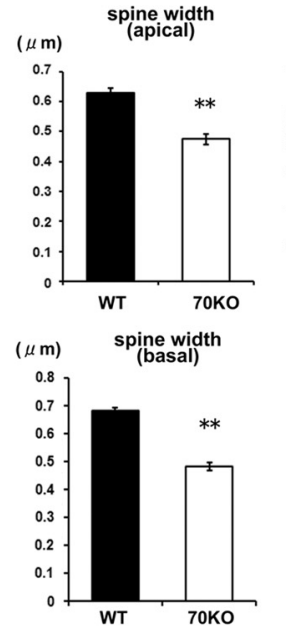
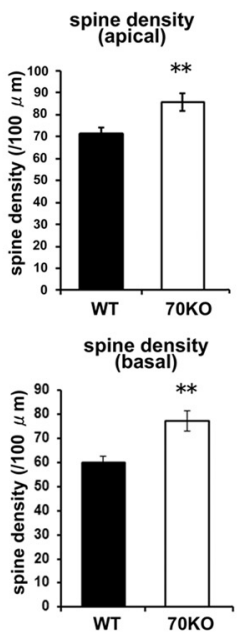
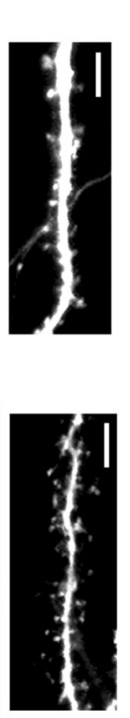

i

i
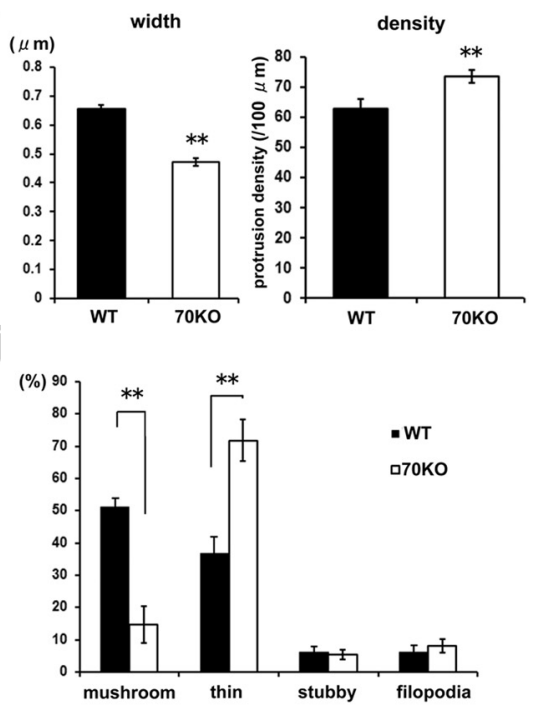

Figure 3. Reduced AMPAR-mediated glutamatergic synaptic transmission with aberrant dendritic spine morphology in PSDZip70KO mice. $\boldsymbol{a}$, Traces of mEPSCs in pyramidal neurons in layer 2/3 of the mPFC slices from WT and PSD-Zip70K0 mice. $\boldsymbol{b}$, The mEPSC frequency was reduced in PSD-Zip70K0 compared with WT neurons $(t=0.284, p=0.009)$, although the mEPSC amplitude was not altered ( $8-12$ weeks of age; WT, $n=12$ from 2 mice; PSD-Zip70K0, $n=15$ from 3 mice). c, Representative traces of EPSCs recorded at -70 and $+40 \mathrm{mV}$ from WT (left) and PSD-Zip70KO slices (right). $\boldsymbol{d}$. The AMPAR/NMDAR ratio was decreased in PSD-Zip70KO neurons ( $t=3.31, p=0.002 ;$ WT, $n=20$ from 3 mice; PSD-Zip70KO, $n=21$ from 4 mice). $\boldsymbol{e}$, Decays of EPSCs at $+40 \mathrm{mV}$ were prolonged in PSD-Zip70KO neurons $(t=35, p=0.047)$. $f$, Golgi staining for pyramidal neurons in layer $2 / 3$ of the ACC in WT and PSD-Zip70K0 mice. Apical (upper) and basal (lower) dendrites are shown. Scale bar, $5 \mu \mathrm{m}$. $g$, Quantification of spine width and density in apical (upper) and basal (lower) dendrites [apical: width, $t=7.29, p<0.001$; density, $t=2.89, p=0.006$; basal, width, $t=10.69, p<0.001$; density: $t=3.27, p=0.002$; mice $=3$, spines $>250$ (size, 727 (density)]. $\boldsymbol{h}$, Typical spine

significantly increased, and the spine density was also increased for PSD-Zip70KO compared with WT neurons (Fig. 3f,g). This aberrant morphology of dendritic spines was also more prominent in primary cultured neurons from the frontal cortex of PSD-Zip70KO mice (Fig. 3h), in which the frequency of thin spines increased, and that of mushroom-type spines decreased (Fig. 3i,j). These data indicate that the PSD-Zip70 deficiency resulted in the formation of tiny-headed thin spines. In accordance with the electrophysiological abnormalities, thin spines of PSD-Zip70KO neurons appear to reflect a functionally immature state of the spine synapse.

PSD-Zip70KO neurons showed fewer mature spine synapses and aberrant Rap2 activation

Western analyses for major synaptic proteins including AMPA- and NMDA-type glutamate receptor subunits revealed no significant differences in their expression levels in the mPFC of PSD-Zip70KO mice (data not shown). We then performed immunocytochemistry for synaptic marker proteins, to compare their localization and clustering status along the dendrites of WT and PSD-Zip70KO neurons. The total signal intensities for the postsynaptic markers PSD-95 and F-actin (stained by phalloidin) were not significantly altered in PSD-Zip70KO neurons; the puncta were smaller than in WT, but their densities were increased (Fig. 4a,b). Conversely, among presynaptic markers, the signal intensity and density of bassoon and vGAT puncta were not altered, but the densities of the synapsin I and vGlut1 puncta were significantly decreased in PSD-Zip70KO neurons (Fig. $4 a, b)$. Bassoon is a presynaptic protein localized to the active zone, synapsin I is an important component of mature presynapses (Mozhayeva et al., 2002; Easley-Neal et al., 2013), and vGlut1 and vGAT are markers for excitatory and inhibitory presynapses, respectively. The formation of inhibitory synapses was considered as normal in PSDZip70KO neurons because there was no alternation of vGAT puncta. The presence of bassoon puncta suggests that the aberrant

morphologies of dissociation cultured neurons expressing EGFP. Scale bar, $5 \mu \mathrm{m}$. $i$, Quantification of spine width and spine density along the dendrites [spine width, $t=9.15, p<$ 0.001 ; density: $t=2.93, p=0.006$; trials $=3$, neurons $\geq 6$, spines $\geq 251$ (size), 547 (density)]. $\boldsymbol{j}$, Classification of spine morphology (Mushroom, $t=4.56, p=0.001$; thin, $t=3.40$, $p=0.006)$. Error bars indicate SEM. Student's $t$ test. ${ }^{*} p<$ $0.05,{ }^{* *} p<0.01$. 


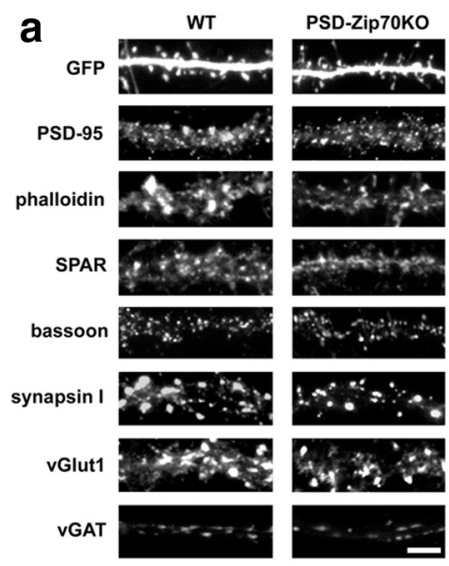

d
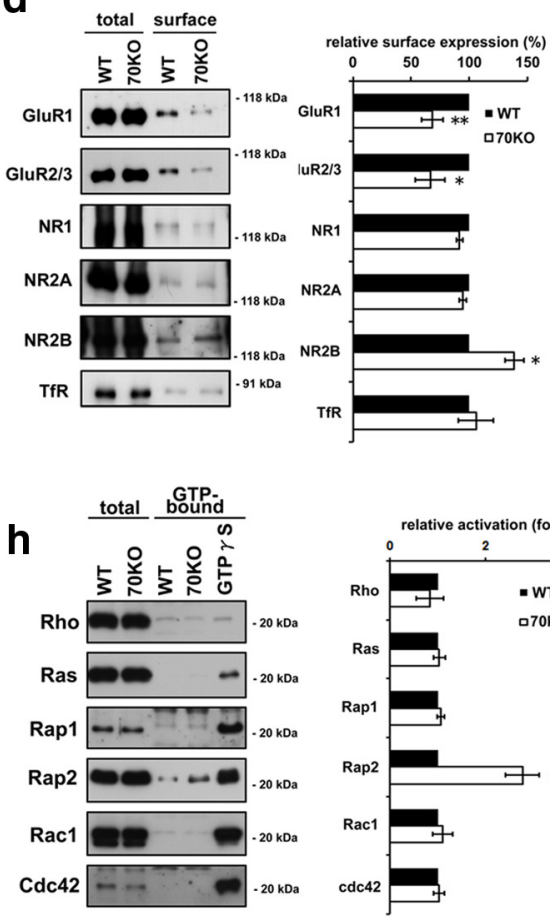
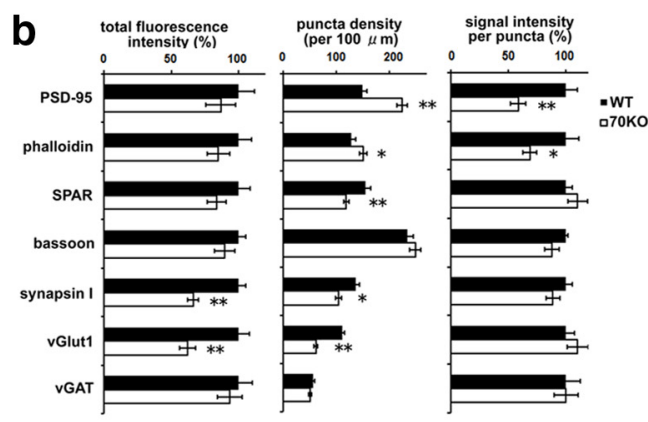

$\mathbf{e}$
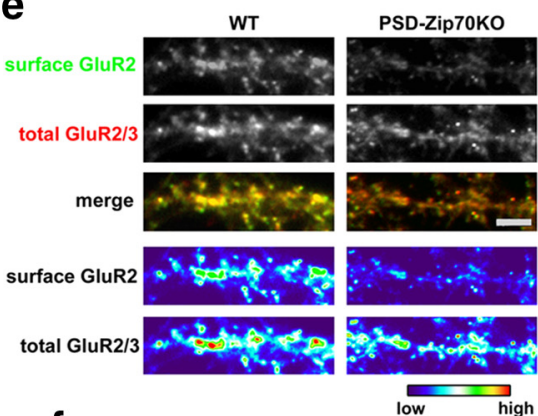

f

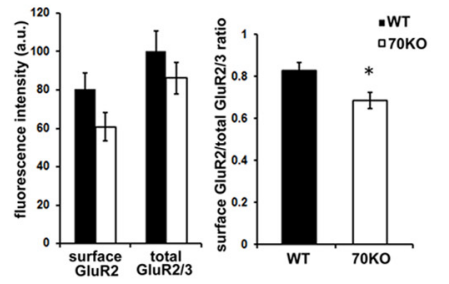

i

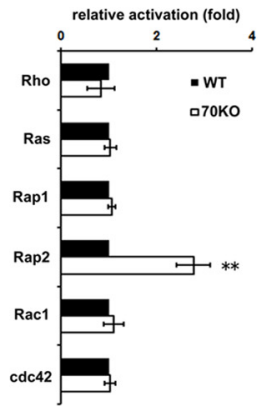

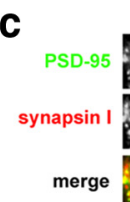

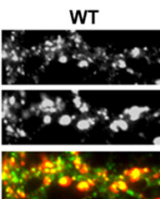

PSD-Zip70KO

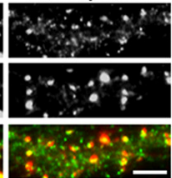

WT
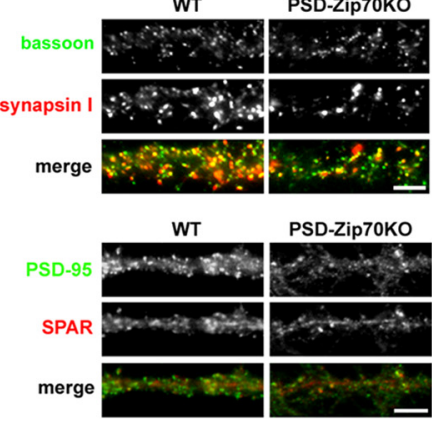

g

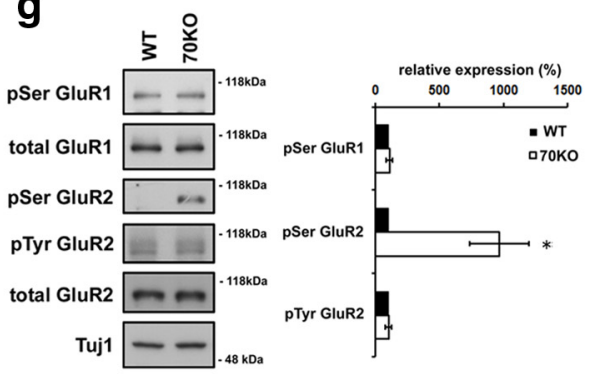

Figure 4. Decrease in mature spine synapses and aberrant activation of Rap2 in PSD-Zip70K0 neurons. $\boldsymbol{a}$, Dendrites of cultured neurons were stained simultaneously for PSD-95 and SPAR (postsynaptic densities), phalloidin (mainly excitatory postsynaptic sites), bassoon (presynaptic terminals), synapsin I (mature presynaptic terminals), vGlut1 (excitatory presynaptic terminals), and vGAT (inhibitory presynaptic terminals). Scale bar, $5 \mu \mathrm{m}$. $\boldsymbol{b}$, Quantified values of the fluorescence intensities (left: synapsin I, $t=4.40, p<0.001 ; \mathrm{vGlut1}, t=3.63, p<0.001$ ), puncta density (middle: PSD-95, $t=6.05, p<0.001$; phalloidin, $t=2.06, p=0.046$; SPAR, $t=3.16, p=0.003$; synapsin $\mathrm{I}, t=2.09, p=0.042$; vGlutl, $t=8.32, p<0.001$ ), and signal intensity per puncta (right:PSD-95, $t=3.34, p=0.002$; phalloidin, $t=2.38, p=0.022$; trials $\geq 3$, neurons $\geq 20$ ). $c$, Dendrites of cultured neurons were costained for the following combinations: PSD-95 and synapsin I (top), bassoon and synapsin I (middle), and PSD-95 and SPAR (bottom). Scale bar, $5 \mu \mathrm{m}$. $\boldsymbol{d}$, The surface expression levels of AMPAR and NMDAR in the frontal cortex were determined by surface biotinylation assay. Quantified values are shown at right. The GluR1 and GluR2/3 subunits for AMPAR were reduced in PSD-Zip70KO frontal cortices (GluR1, $t=3.16, p=0.006 ;$ GluR2/3, $t=2.64$, $p=0.018 ; \mathrm{NR} 2 \mathrm{~B}, t=3.91, p=0.017 ; n \geq 3)$. TfR, Transferrin receptor as a loading control.e, The surface GluR2 was labeled with antibody against the extracellular domain before permeabilization (green), and the total GluR2/3 (red) was labeled after permeabilization. Fluorescence intensity of surface GluR2 is shown as a heat map (bottom). Scale bar, $5 \mu \mathrm{m} . \boldsymbol{f}$, Quantified values of the fluorescence intensities (left) and the ratio of surface GluR2 to total GluR2/3 intensities (right; $t=2.60, p=0.014$; trials $\geq 3$, neurons $\geq 16$ ). $\boldsymbol{g}$, Phosphorylated AMPAR subunits were detected using specific antibodies for phospho-Ser845 of GluR1, and phospho-Ser880 and phospho-Tyr869/873/876 of GluR2. Cell lysates of the mPFC from WT and PSD-Zip70K0 mice were analyzed by Western blotting (pSer GluR2, $t=3.41, p=0.007 ; n \geq 3$ ). $\boldsymbol{h}$,Small GTPase activity assays were performed by pull-down assay using affinity beads specific for the GTP-bound form. Quantified values are shown at right. Rap2-specific activation was observed in the lysate from PSD-Zip70K0 frontal cortices (Rap2, $t=4.06, p=0.007 ; n \geq 3$ ). $\boldsymbol{i}$, Rap2 activity assays were performed using brain lysates prepared from the PFC, striata (Str), and hippocampi (Hipp) of 8-week-old male WT or PSD-Zip70K0 mice (PFC, $t=8.06, p<0.001$ : Str, $t=11.94, p<0.001$; Hipp, $t=3.12, p=0.014$ ). Error bars indicate SEM. Student's t test. ${ }^{*} p<0.05,{ }^{* *} p<0.01$

morphological dendritic spines form synapses in PSD-Zip70KO neurons. The decreases in synapsin I and vGlutl puncta may indicate that nascent excitatory spine synapses do not mature. Indeed, nearly half of the PSD-95 or bassoon puncta were not accompanied by synapsin I puncta in PSD-Zip70KO neurons (Fig. 4c). These results supported the notion that the PSD-Zip70 deficiency caused defects in the maturation of nascent spine synapses, in correlation with a decrease in mushroom-type spines (Fig. $3 j$ ) and with the electrophysiological alteration in the mEPSC frequency, but not amplitude (Fig. 3b).

Further, surface biotinylation assay revealed that the surface expressions of GluR1 and GluR2/3, AMPA-type glutamate receptor subunits, were decreased by $>30 \%$ in PSD-Zip70KO compared with WT neurons (GluR1, $68.2 \pm$ 9.5\%; GluR2/3, $66.5 \pm$ 

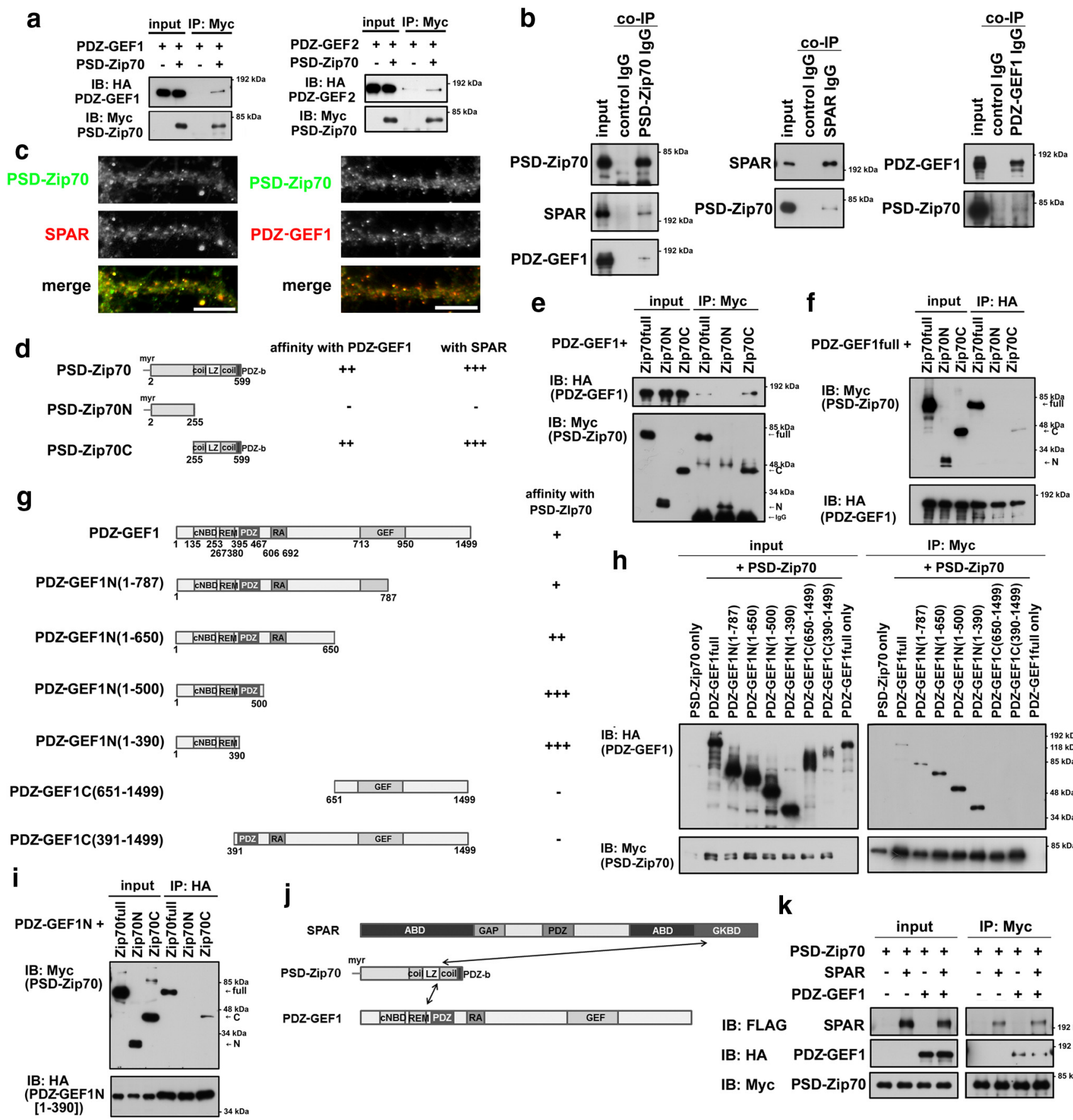

PDZ-GEF1 $\square$ CNBGREMMPDZ $\mid$ RA
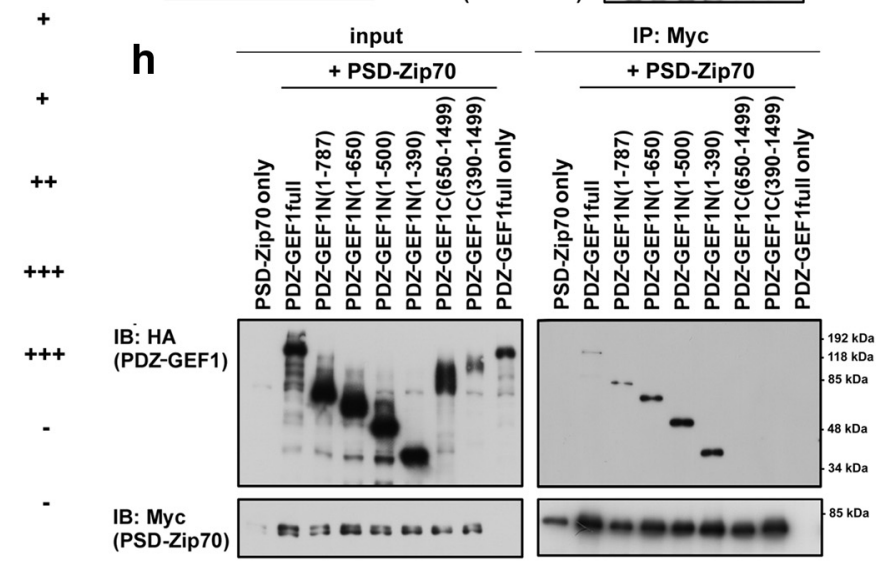

k

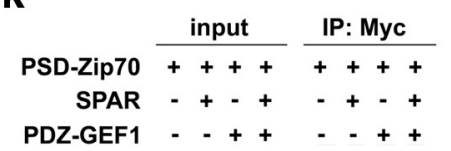

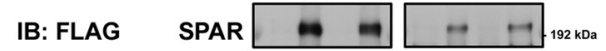

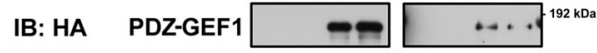
IB: Myc PSD-Zip70

Figure 5. PSD-Zip70 interacts with both SPAR and PDZ-GEF1. $\boldsymbol{a}$, HEK293T cells were transfected with Myc-tagged PSD-Zip70 in combination with HA-tagged PDZ-GEF1 or PDZ-GEF2, and co-IP assays were performed using cell lysates with an anti-Myc antibody. $\boldsymbol{b}$, co-IP assay was performed using anti-PSD-Zip70, anti-SPAR, and anti-PDZ-GEF1 antibodies with lysates from mouse cortices. Endogenous SPAR and PDZ-GEF1 were co-immunoprecipitated with endogenous PSD-Zip70. The proteins in the immunoprecipitants were detected by Western blotting with the indicated antibodies. c, PSD-Zip70 was colocalized with SPAR (left) and PDZ-GEF1 (right) in cultured WT neurons. Scale bar, $10 \mu \mathrm{m}$. $\boldsymbol{d}$, Schematic representation of the truncated fragments of PSD-Zip70. The $\mathrm{N}$-terminal myristoylation motif, coiled-coil (coil) domain, leucine zipper (LZ) domain, and PDZ-binding motif are shown. Numbers are amino acid positions. $\boldsymbol{e}, \boldsymbol{f}$, HEK293T cells were transfected with Myc-tagged PSD-Zip70 fragments in combination with HA-PDZ-GEF1full, and co-IP assays were performed using cell lysates with anti-Myc $(\boldsymbol{e})$ or anti-HA $(\boldsymbol{f})$ antibodies. $\boldsymbol{g}$, Schematic representation of the truncation series of PDZ-GEF1. The pseudocyclic-nucleotide binding domain (CNBD), Ras exchange motif (REM), PDZ domain, Ras-association (RA) domain, and RapGEF (GEF) domain are shown. $\boldsymbol{h}$, HEK293T cells were transfected with various HA-tagged PDZ-GEF1 fragments in combination with Myc-tagged PSD-Zip70full, and co-IP assays were performed using the cell lysates with anti-Myc antibody. $\boldsymbol{i}$, HEK293T cells were transfected with Myc-tagged PSD-Zip70 fragments in combination with HA-tagged PDZ-GEF1N(1-390), and co-IP assays were performed using cell lysates with anti-HA antibody. $\boldsymbol{j}$, Schematic diagram of the interactions among PSD-Zip70, SPAR, and PDZ-GEF1. $\boldsymbol{k}$, co-IP assay was performed in HEK293T cells with Myc-PSD-Zip70 in combination with HA-PDZ-GEF1 and/or FLAG-SPAR. The co-immunoprecipitated proteins with Myc-PSD-Zip70 were analyzed.

12.7\%; Fig. 4d). In contrast, the surface expression of NR2B was increased in PSD-Zip70KO neurons $(138 \pm 8.10 \%)$, although those of NR1 and NR2A were unchanged (Fig. 4d). The increased surface expression of NR2B appeared to be consistent with the electrophysiological data shown in Figure $3 e$. We next examined the distribution of surface GluR2 along the dendrites. The surface GluR2/total GluR2/3 ratio was significantly decreased in PSDZip70KO neurons (Fig. 4e,f). Previous studies showed that the 


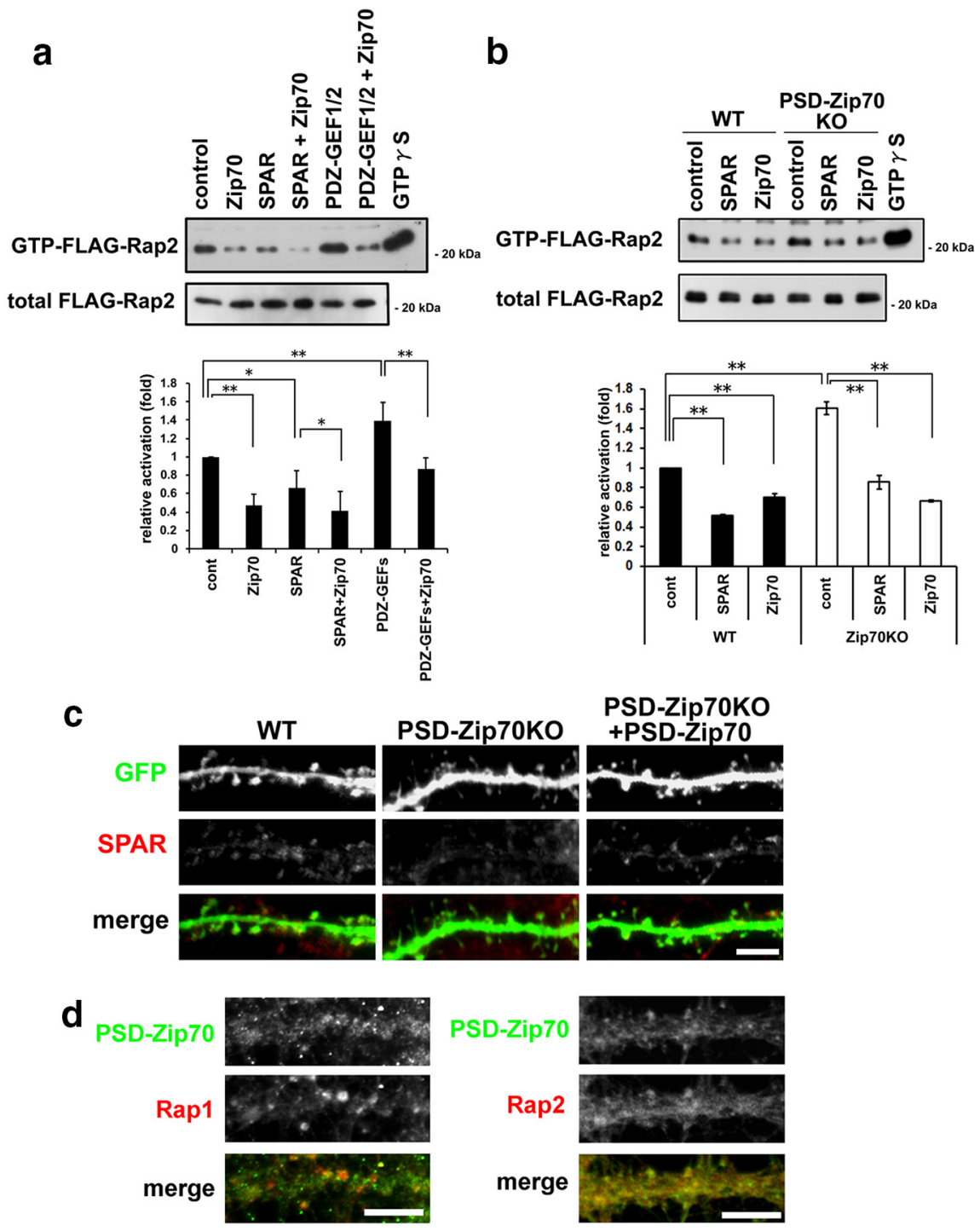

Figure 6. PSD-Zip70 modulates Rap2 activity via SPAR- and PDZ-GEF1. $\boldsymbol{a}$, Rap2 activity assay was performed in cultured WT neurons. Cultured neurons were cotransfected with exogenous FLAG-tagged Rap2 in combination with the indicated expression plasmids. Total and GTP-bound exogenous Rap2 were detected using anti-FLAG antibody. SPAR and PSD-Zip70 cooperatively suppressed the Rap2 activity. The RapGEF activity of PDZ-GEF1 and PDZ-GEF2 activated Rap2, and PSD-Zip70 coexpression suppressed the PDZ-GEF-dependent Rap2 activation. Quantified values are shown in the lower graph $\left(F_{(5,30)}=31.35, p<0.001 ; n=\right.$ 6). $\boldsymbol{b}$, Rap2 activity assay was performed in cultured neurons. SPAR or PSD-Zip70 expression rescued the aberrant Rap2 activation in PSD-Zip70KO neurons. Quantified values are shown in the lower graph $\left(F_{(5,18)}=68.28, p<0.001 ; n=4\right)$. c, Exogenously expressed PSD-Zip70 rescued the proper targeting of SPAR to spines in the PSD-Zip70KO neurons. Scale bar, $5 \mu \mathrm{m}$. $\boldsymbol{d}$, Dendrites of cultured WT neurons were stained for the following combinations: PSD-Zip70 and Rap1 (left) or PSD-Zip70 and Rap2 (right). Scale bar, $10 \mu \mathrm{m}$. Error bars indicate SEM. One-way ANOVA with Tukey-Kramer post hoc test. ${ }^{*} p<0.05,{ }^{* *} p<0.01$.

phosphorylation of AMPARs at their cytoplasmic terminus is correlated with their surface expression at postsynaptic sites (Wang et al., 2005). We found that the phosphorylation of GluR2 at $\mathrm{S} 880$, which is correlated with its internalization in response to long-term depression (LTD) induction (Lu and Roche, 2012), was markedly elevated in PSD-Zip70KO neurons (Fig. 4g).

The Rho, Ras, and Rap small GTPases play important roles in spine formation and synaptic maturation (Bonhoeffer and Yuste, 2002; Zhu et al., 2005; Lee et al., 2011). Among them, we found that the activity of Rap 2 was specifically upregulated in the frontal cortex of PSD-Zip70KO mice (2.77 \pm 0.32 -fold activation compared with WT; Fig. $4 h, i)$. The Rap 2 activation was also observed in the hippocampus ( $1.97 \pm 0.44$-fold) and the striatum (1.83 \pm 0.10 -fold; Fig. 4i). A similar upregulation of Rap2 activity was found in cultured PSD-Zip70KO neurons prepared from the frontal cortex (2.56 \pm 0.16 -fold; data not shown). The Rap2 activity is reported to be a critical regulator of AMPAR internalization in response to LTD-inducing stimuli (Zhu et al., 2005; Fu et al., 2007).

\section{PSD-Zip70 interacts with both SPAR} and PDZ-GEF1, and modulates the Rap2 activity

The activity of Rap 2 is regulated by a balance between the GEF (guanine nucleotide exchange factor) and GAP (GTPase-activating protein) activities. We previously found that PSD-Zip70 interacts with SPAR, a spineenriched RapGAP (Maruoka et al., 2005). We therefore presumed that the activities of GEF and/or GAP for Rap2 would be dysregulated in PSD-Zip70KO neurons. The localization of SPAR along the dendrites was diffuse along the dendrites of PSDZip70KO neurons (Fig. $4 a-c$ ), even though SPAR's expression level was not altered (Fig. $4 a, b)$. These results suggested that the PSDZip70 deficiency weakened SPAR's targeting to the spines, and might have altered SPAR's RapGAP activity.

To elucidate the mechanism underlying the specific activation of Rap2, we next examined possible interactions between PSD-Zip70 and reported RapGEFs, because SPAR possesses catalytic activity to both Rap1 and Rap2. Among the RapGEFs examined, we identified PDZGEF1 and PDZ-GEF2 as novel PSDZip70-interacting proteins (Fig. 5a). PDZ-GEF1 is reported to be more highly expressed than PDZ-GEF2 in the brain (Lee et al., 2011). We confirmed that endogenous PSD-Zip70 interacted with both SPAR and PDZ-GEF1 in lysates prepared from the frontal cortex (Fig. 5b). PSD-Zip70 showed the predominant expression in excitatory pyramidal neurons, which possess numerous spines on the dendrites in cultured cortical as well as hippocampal neurons (Fig. $5 c$; Konno et al., 2002). PSD-Zip70 was colocalized with SPAR and with PDZ-GEF1 as puncta at mainly dendritic spines in WT neurons (Fig. $5 c$ ).

Next, we analyzed the interaction sites for PSD-Zip70 and PDZ-GEF1 in heterologous HEK293T cells. The results revealed that the C-terminal region of PSD-Zip70 and the $\mathrm{N}$-terminal region of PDZ-GEF1 were required for their interaction (Fig. $5 d-j$ ). PDZ-GEF1 lacking the C terminus exhibited a high affinity for PSD-Zip70 compared with the fulllength form, suggesting that the $C$ terminus of PDZ-GEF1 suppresses its interaction with PSD-Zip70 (Fig. 5h). Furthermore, PDZ-GEF1 did not compete with SPAR for the PSDZip70 binding (Fig. $5 k$ ). These results together suggested that PSD-Zip70 forms a ternary complex with SPAR and PDZGEF1 at the postsynaptic site. 
a

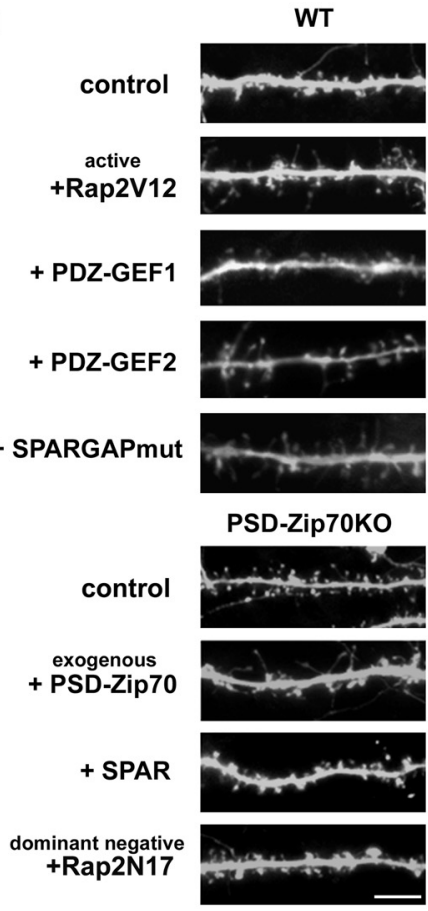

d
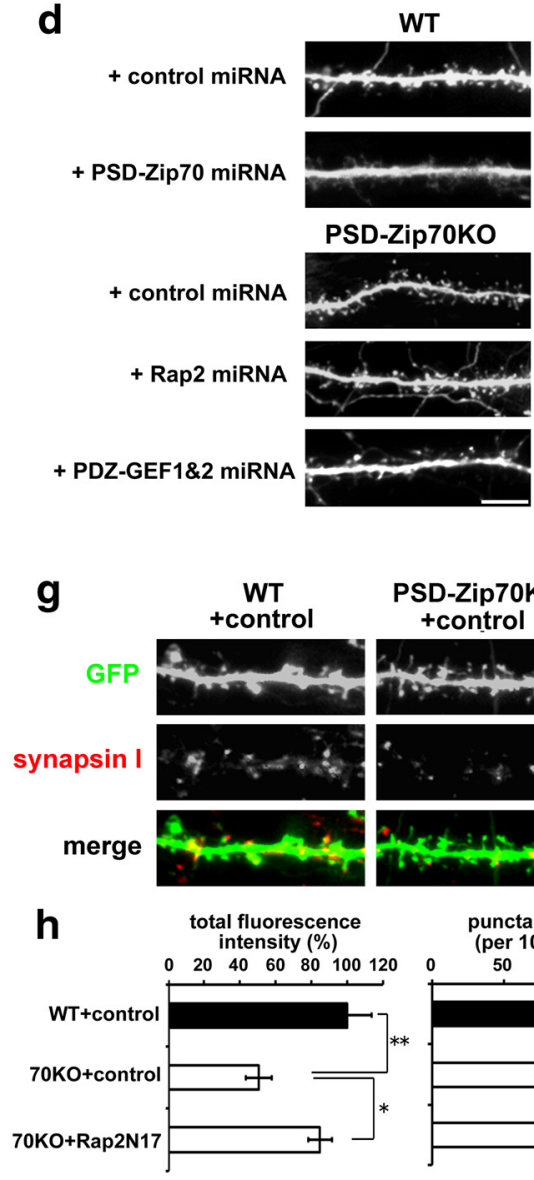

PSD-Zip70Ko
+ control

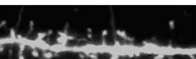

b
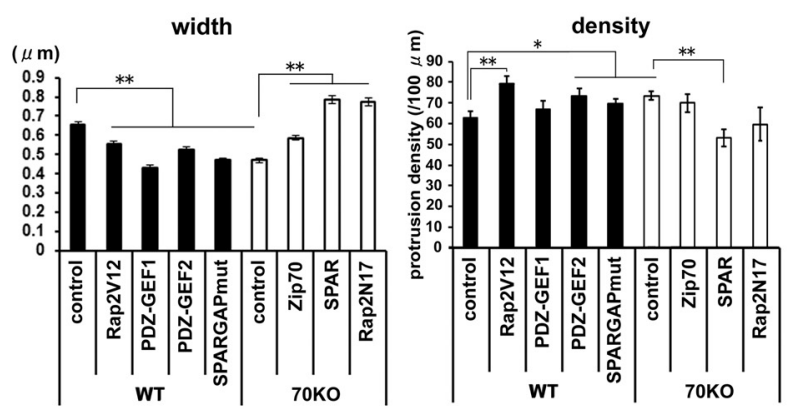

C

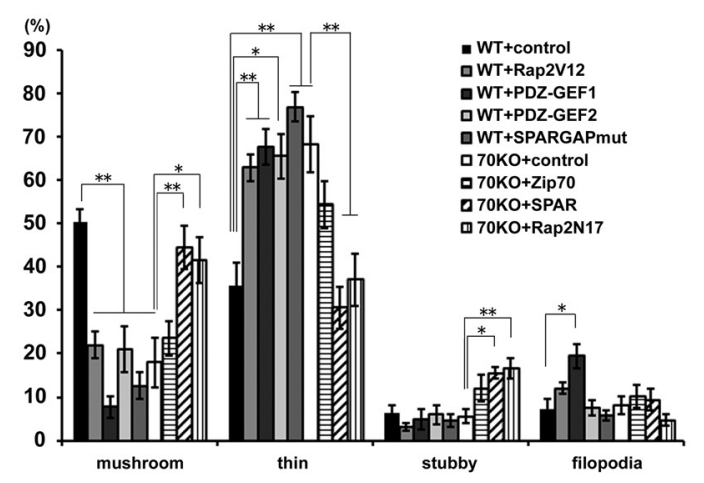

e
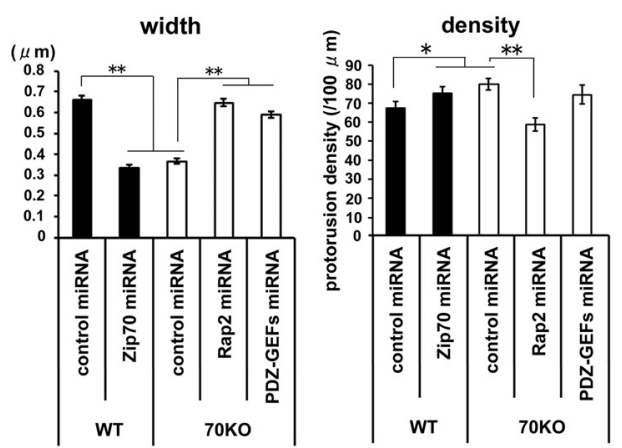

f $(\%)$
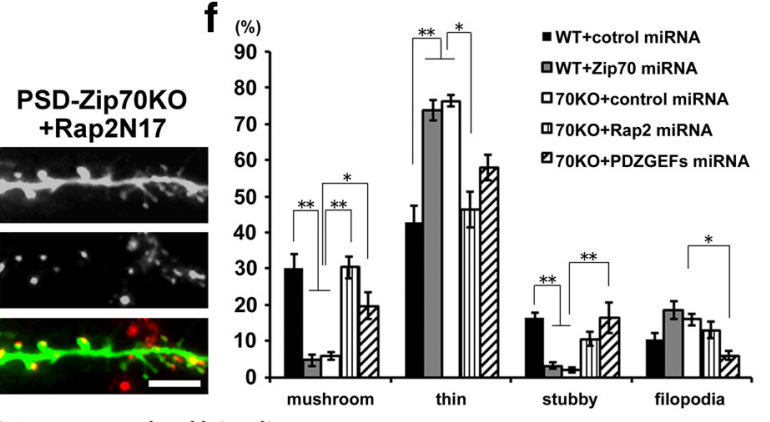

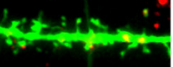

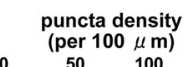

signal intensity

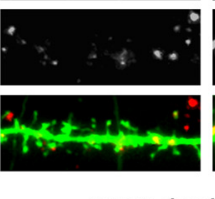

$50 \quad 100$

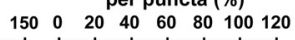
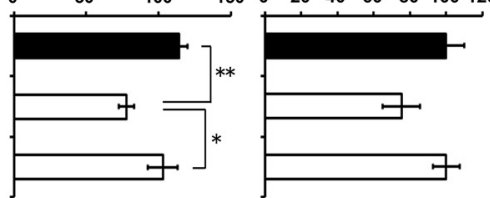

Figure 7. PSD-Zip70 regulates spine morphology via modulation of the Rap2 activity. $\boldsymbol{a}$, Typical spine morphologies of cultured neurons expressing EGFP with the indicated expression vectors. Forced expression of PDZ-GEF1, PDZ-GEF2, or an active form of Rap2 (Rap2V12) induced the formation of spines with smaller heads in WT neurons. Forced expression of an inactive form of Rap2 (Rap2N17), SPAR, or exogenous PSD-Zip70 recovered the spine head size in PSD-Zip70KO neurons. Scale bar, $5 \mu \mathrm{m}$. $\boldsymbol{b}$, Quantification of protrusion width (trials $\geq 3$, neurons $\geq 6$, spines $\geq 235$; $F_{(8,25)}=68.85, p<0.001$ ), and density (trials $\geq 3$, neurons $\geq 6$, spines $\left.\geq 383 ; F_{(8,194)}=7.00, p<0.001\right)$. $c$, Classification of spine morphology (Mushroom, $F_{(8,58)}=9.19, p<0.001 ;$ thin, $F_{(8,58)}=10.04, p<0.001$; stubby, $F_{(8,58)}=5.39, p<0.001$; filopodia, $F_{(8,58)}=4.10, p<0.001$, trials $\geq 3$, neurons $\geq 6$, spines $\left.\geq 235\right)$. $\boldsymbol{d}$, Typical spine morphologies of dissociation cultured neurons transfected with the indicated miRNA-mediated knockdown vectors. Depletion of PSD-Zip70 induced the formation of thin-type spines in WT neurons, similar to PSD-Zip70K0 neurons. Depletion of Rap2 or PDZ-GEFs by miRNA rescued the spine-formation defects in PSD-Zip70K0 neurons. Scale bar, $5 \mu \mathrm{m}$. $\boldsymbol{e}$, Quantification protrusion width (trials $\geq 3$, (Figure legend continues.) 
We then examined the effect of PSD-Zip70's interactions with SPAR and/or PDZ-GEF1 on the Rap2 activity in neurons. The SPAR suppressed the coexpressed FLAG-Rap2 activity by its RapGAP activity, and the coexpression of SPAR and PSD-Zip70 cooperatively suppressed the Rap2 activity in WT neurons (Fig. 6a). Further, expression of PSD-Zip70 or SPAR could suppress the aberrant Rap2 activation in PSD-Zip70KO neurons (Fig. 6b). The impaired spine targeting of SPAR in PSD-Zip70KO neurons was reversed by the moderate expression of exogenous PSDZip70 (Fig. 6c). These results suggested that PSD-Zip70 assists the spine targeting of SPAR and enhances the efficiency of SPAR's RapGAP activity.

PDZ-GEF1/2 activated Rap2 by its RapGEF activity, and its coexpression with PSD-Zip70 suppressed the PDZ-GEF-dependent Rap2 activation (Fig. 6a). The suppressive effect of PSD-Zip70 on the GEF activity of PDZ-GEF1/2 for Rap1/2 was also observed in HEK293T cells (data not shown). Further, we found that PSD-Zip70 and Rap2 were colocalized as puncta along the dendrites and spines, whereas Rap1 was localized as prominent puncta without PSDZip70 (Fig. 6d). Thus, the Rap2-specific activation in PSD-Zip70KO neurons may be related to the spatial association of PSD-Zip70 with SPAR and PDZ-GEF1 as a ternary complex close to Rap2 in the postsynapse.

\section{PSD-Zip70 regulates spine synapse maturation by modulating the Rap2 activity}

We then investigated the effect of Rap2 activity on the spine morphology in WT and PSD-Zip70KO neurons. The forced expression of PDZ-GEF1 or PDZ-GEF2 reduced the size of the spine head and altered the spine shape from mushroom to thin in WT neurons (Fig. 7a-c). The moderate expression of a constitutively active form of Rap2 (Rap2V12) using a low-dose expression plasmid caused similar results when coexpressed with PDZ-GEFs (Fig. 7a-c). The Rap2-dependent effects on spine morphology were confirmed by expressing a GAP-mutated SPAR that functions as a dominant-negative form with respect to SPAR's RapGAP activity. In contrast, the expression of Rap2N17 (an inactive form of Rap2; Rap2DN), SPAR, or exogenous PSDZip70 recovered the spine morphology in PSD-Zip70KO neurons (Fig. $7 a-c$ ). We confirmed that depleting PSD-Zip70 by miRNA-mediated knockdown in WT neurons mimicked the spine-morphology phenotype of PSD-Zip70KO neurons (Fig. $7 d-f$ ). In contrast, depleting all of the Rap2 subtypes (Rap2a, b, and c) or both PDZ-GEF1 and PDZ-GEF2 could recover the spine-morphology defects of PSD-Zip70KO neurons (Fig. 7d-f). We further confirmed that the decrease in synapsin I puncta along the dendrites of PSD-Zip70KO neurons was recovered by expressing Rap2N17 (Fig. 7g,h). Thus, our results clearly suggest that the Rap2 activation in PSD-Zip70KO neurons causes synaptic dysfunction accompanied by impaired spine maturation.

\footnotetext{
$\leftarrow$

(Figure legend continued.) neurons $\geq 6$, spines $\geq 282 ; F_{(4,1610)}=131.10, p<0.001$ ) and density along the dendrites (trials $\geq 3$, neurons $\geq 6$, spines $\geq 489 ; F_{(4,92)}=9.321, p<$ 0.001). $f$, Classification of spine morphology (Mushroom, $F_{(4,37)}=13.31, p<0.001$; thin, $F_{(4,37)}=12.11, p<0.001 ;$ stubby, $F_{(4,37)}=11.95, p<0.001$; filopodia, $F_{(4,37)}=4.93, p=$ 0.003 ; trials $\geq 3$, neurons $\geq 6$, spines $\geq 282)$. $\boldsymbol{g}$, Dendrites of cultured neurons transfected with EGFP and Rap2N17 or control (empty vector) were stained for synapsin I. Scale bar, $5 \mu \mathrm{m}$. $\boldsymbol{h}$, Quantified values of the fluorescence intensities (left; $F_{(2,33)}=8.37, p=0.001$ ), puncta density (middle; $F_{(2,33)}=8.18, p=0.001$ ), and signal intensity per puncta (right; $F_{(2,33)}=$ $2.75, p=0.120$ ) for synapsin I (trials $\geq 3$, neurons $\geq 9$ ). Error bars indicate SEM. One-way ANOVA with Tukey-Kramer post hoc test.
}

\section{Rescue of behavioral abnormalities by Rap 2 inactivation in the mPFC}

Because the suppression of Rap2 activation almost recovered the spine-morphology defects in PSD-Zip70KO neurons, we examined whether inactivating Rap2 in the MPFC could ameliorate the behavioral abnormalities seen in the PSD-Zip70KO mice. By in utero electroporation, a plasmid-expressing Rap2N17 (Rap2DN) was bilaterally transfected into the MPFC of PSD-Zip70KO mice (Fig. 8a). The Rap2N17-transfected PSD-Zip70KO mice exhibited less anxiety-related behavior in the elevated plus maze test (Fig. $8 b$ ) and improved spatial working memory in the Y-maze test (Fig. 8c). These results indicated that the PSD-Zip70 deficiency in the mPFC was responsible for the defects in working memory and enhanced anxiety, through Rap2 activation. In contrast, the cognition assessed by the novel object recognition test in PSD-Zip70KO mice was hardly affected by Rap2N17 transfection into the mPFC (Fig. 8d).

\section{Discussion}

Regulation of synaptic maturation is an essential process for the neuronal system and dysregulation is involved in the pathophysiology of psychiatric and neurodevelopmental disorders. However, the cellular and molecular mechanisms remain unclear. Our study provides evidence that PSD-Zip70 regulates spine morphology and glutamatergic synapse maturation by modulating the Rap2 activity.

Previous reports demonstrated that Rap2 is a critical regulator of the surface AMPAR-mediated synaptic transmission (Fu et al., 2007; Ryu et al., 2008; Lee et al., 2011). Here we demonstrated that PSD-Zip70 deficiency specifically activates Rap2 (Fig. 4). We found that SPAR and PDZ-GEF1, the PSD-Zip70-interacting RapGAP and RapGEF respectively, reciprocally regulate the Rap2 activity, and that the specificity for Rap2 is because of spatial interaction of the PSD-Zip70/SPAR/PDZ-GEF1 ternary complex with Rap2 in dendritic spines (Fig. 5). Rap2 requires lipid modifications such as farnesylation and palmitoylation for its activity and cellular localization (Uechi et al., 2009). We found that the synaptic targeting of SPAR was significantly impaired in PSDZip70KO neurons (Figs. 4, 6). PSD-Zip70 may assist in targeting SPAR close to the lipid-modified Rap2 in the postsynapse, because PSD-Zip70 is targeted to the postsynaptic membrane by its $\mathrm{N}$-terminal myristoylation (Konno et al., 2002). So far, the mechanisms for the specific activation of Rap1 or Rap2 have not been elucidated, because most of their known GAPs and GEFs are reported to have redundant catalytic activities for both Rap1 and Rap2 (de Rooij et al., 1999; Pak et al., 2001). Our results revealed a novel molecular mechanism of the synaptic regulation mediated specifically by Rap2 activity.

Rap2 activation increased the density of tiny-headed thin spines in neurons (Figs. 3, 7). In correlation with the morphological defects, the AMPAR-mediated glutamatergic synaptic transmission was impaired in PSD-Zip70KO neurons (Figs. 3, 4). Our data indicated that the PSD-Zip70 deficiency caused excitatory spine synapse-maturation defects and increases in nonfunctional immature synapses like silent synapses. This hypothesis was supported by the results of predominance of NR2B-containing immature-type NMDARs in PSD-Zip70KO neurons, as observed in silent synapses (Isaac et al., 1995; Rumpel et al., 1998; Renger et al., 2001; Fig. 3), and increase in synapsin I-lacking immature synapses (Fig. 4). These data indicated that the maturation of nascent spine synapses was impaired in PSD-Zip70KO neurons.

The synaptic dysfunction caused by PSD-Zip70 deficiency in the PFC caused behavioral abnormalities. The reduced mature 


\section{a}

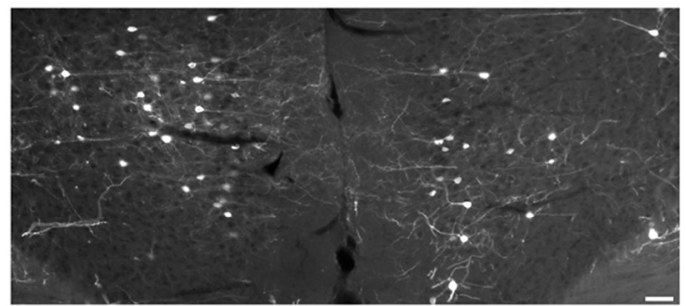

b

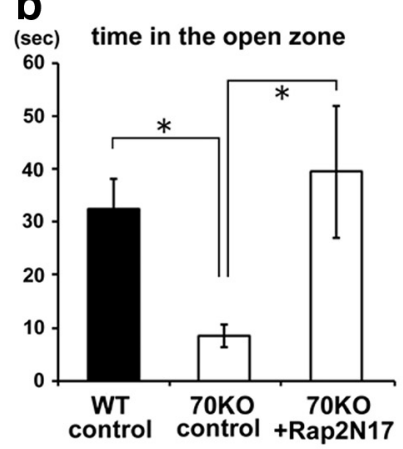

C number of total entries

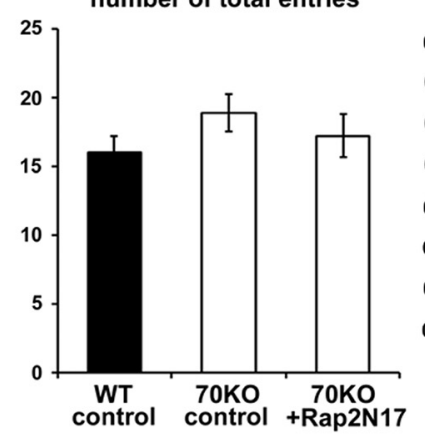

d time in the familiar zone (sec)

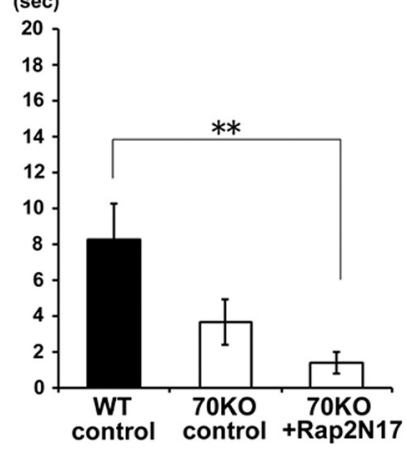

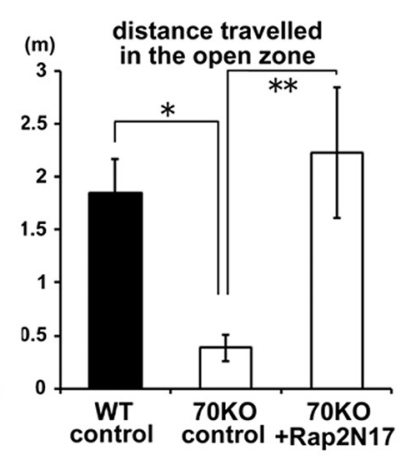

alternation

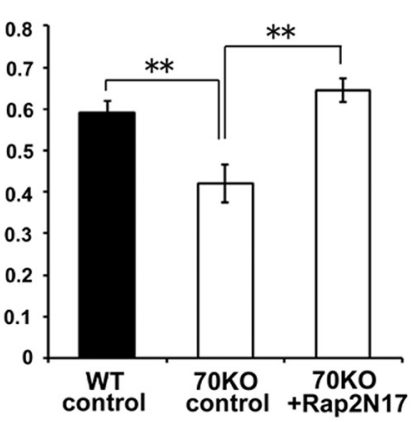

time in the novel zone

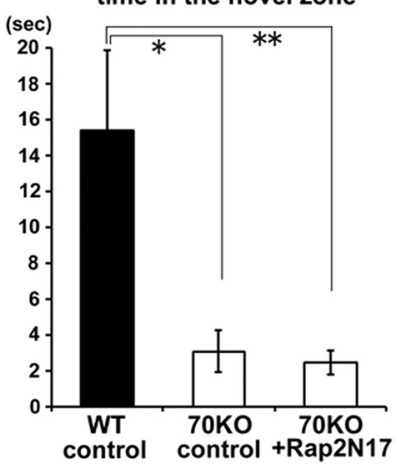

Figure 8. Rescue of impaired spine maturation by the inactivation of Rap2 in PSD-Zip70K0 neurons. The mPFC of PSD-Zip70K0 mice was transfected with Rap2N17, and the behavioral properties were compared with those of WT and PSD-Zip70KO mice. $\boldsymbol{a}$, Representative coronal images of a 10-week-old mouse brain. The mouse had been transfected with EGFP and Rap2N17 expression plasmids in the MPFC using in utero electroporation at E14.5. Scale bar, 50 $\mu \mathrm{m} . \boldsymbol{b}$, Elevated plus maze test. PSD-Zip70KO mice exhibited shorter durations and track lengths into the open arms (open zone). The tendency to avoid entry to the open arms was ameliorated in the Rap2N17-transfected PSD-Zip70K0 mice. (time, $F_{(2,25)}=3.75, p=0.038$; distance, $F_{(2,25)}=5.11, p=0.014 ;$ WT, $n=10 ;$ PSD-Zip70K0, $n=9$; PSDZip70KO + Rap2N17, $n=9$ ). One-way ANOVA and Fisher's protected least significant difference test was performed as post hoc test. c, Y-maze test. Rap2N17-transfected PSD-Zip70K0 mice displayed normal locomotor activity (left) and a reversed alternation score from that of the PSD-Zip70K0 mice (right; alternation, $F_{(2,25)}=9.81, p=0.001$; WT, $n=10 ;$ PSD-Zip70K0, $n=9$; PSD-Zip70KO + Rap2N17, $n=9$ 9). One-way ANOVA with Tukey-Kramer post hoc test. $\boldsymbol{d}$,

synapse numbers decreases synaptic transmission efficiency, and the alteration of NMDAR subunit composition should also affect NMDAR-mediated synaptic plasticity, which is closely involved in learning and memory. The dorsolateral prefrontal cortex (dlPFC) and mPFC in humans and the dorsal mPFC including the ACC and prelimbic cortex in rodents are known to play important roles in higher-order executive functions such as working memory, cognition, and emotional control (Levy et al., 2000; Dumitriu et al., 2010; Etkin et al., 2011; Euston et al., 2012). Recent neuroimaging analyses implicate hypofrontality, which means hypofunction of the PFC observed by decreased cerebral blood flow (CBF) during a test of executive function, as an important factor underlying the pathophysiology of psychiatric and neurodevelopmental disorders (Zang et al., 2005; Ragland et al., 2007; Kinou et al., 2013). Working memory is thought to provide the temporal storage of information, the retrieval of temporal information from long-term memory, and the manipulation of the information in performing mental tasks (Wild-Wall et al., 2011). The recognition memory performance assessed by the novel object recognition test also involves the PFC (Miller et al., 1996). These cognitive abilities are impaired in schizophrenia, depression, and ASD, and affect the pathophysiological traits of these disorders. Enhanced anxiety is frequently observed, not only in anxiety disorders, but also in mood disorders including depression. One hypothesis is that impaired working memory and/or cognition results in a failure to suppress the anxiety evoked by novel information (Holmes and Wellman, 2009). Our results indicated that $\mathrm{PSD}$-Zip70 deficiency caused hypofunction of the mPFC through defects in glutamatergic synaptic transmission by aberrant Rap 2 activation, which leads the behavioral defects (Figs. 2-4). This scenario was strongly supported by in vivo experiments, in which Rap2 inactivation in the mPFC of PSDZip70KO mice ameliorated the behavioral defects in working memory and enhanced anxiety (Fig. 8). On the other hand, cognition assessed by the novel object recognition test was not rescued by the approach (Fig. 8). This task requires memory consolidation and retrieval as well as recognition of the difference in the objects, and the hippocampus is also important for the object recognition memory paradigm (Antunes and Biala, 2012). Because PSD-Zip70 is highly expressed in the hippocampus in addition to the PFC (Konno et al., 2002), it is not surprising that this behavioral defect was not affected by the Rap 2 inactivation, which only occurred in the mPFC. It is also characterized by the normal spontaneous locomotion and sociability in PSDZip70KO mice (Fig. 2). Recently, RapGEFs, EPAC2 and PDZGEF2, were reported as susceptibility candidates for autism and schizophrenia (Bacchelli et al., 2003; Chen et al., 2006). Accumulating reports indicate that regulation of Rap activity is involved in psychiatric and neurodevelopmental disorders. Combined with our results, the PSD-Zip70KO mice may be a useful model for analyzing the mechanisms underlying Rap2-dependent synaptic dysfunction in the $\mathrm{mPFC}$ and its associated behavioral traits, to improve our understanding of psychiatric and neurodevelopmental disorders.

It is noteworthy that PSD-Zip70 (Lzts1) was identified as a tumor suppressor located on chromosome 8p22 (Ishii et al.,

$\leftarrow$

Novel object recognition (test. Rap2N17-transfected PSD-Zip70KO mice exhibited a lower preference to access both novel and familiar objects, similar to PSD-Zip70KO mice (familiar, $F_{(2,25)}=5.81, p=0.007 ;$ novel, $F_{(2,25)}=7.11, p=0.004 ;$ WT, $n=10 ;$ PSD-Zip70K0, $n=9$; PSD-Zip70K0 + Rap2N17, $n=9)$. Error bars indicate SEM. ${ }^{*} p<0.05,{ }^{* *} p<0.01$. 
1999; Vecchione et al., 2001), and that the 8p region is well known to be related to susceptibility to psychiatric disorders as well as cancer (Tabarés-Seisdedos and Rubenstein, 2009). Genomewide linkage studies provided evidence that the 8 p22 region influences susceptibility to psychiatric phenotypes such as schizophrenia, bipolar disorder, depression, and autism (Kendler et al., 1999; Park et al., 2004; Zubenko et al., 2004; Cheng et al., 2006; Papanikolaou et al., 2006). Shyn et al. (2011) reported that an SNP (single-nucleotide polymorphism) in 8p21.3 flanking Lzts 1 gene is associated with depression. Although the association of the Lzts1 (PSD-Zip70) gene with psychiatric and neurodevelopmental disorders is a subject for future study, the present results may provide a fundamental mechanism for PSD-Zip70's role in the pathophysiology of these disorders.

\section{Conclusion}

Our findings reveal essential roles of PSD-Zip70 in the mPFC in working memory, cognition, and emotional control, through modulation of the Rap2 activity, which regulates glutamatergic spine synapse maturation.

\section{References}

Antunes M, Biala G (2012) The novel object recognition memory: neurobiology, test procedure, and its modifications. Cogn Process 13:93-110. CrossRef Medline

Bacchelli E, Blasi F, Biondolillo M, Lamb JA, Bonora E, Barnby G, Parr J, Beyer KS, Klauck SM, Poustka A, Bailey AJ, Monaco AP, Maestrini E; International Molecular Genetic Study of Autism Consortium (IMGSAC) (2003) Screening of nine candidate genes for autism on chromosome $2 \mathrm{q}$ reveals rare nonsynonymous variants in the cAMP-GEFII gene. Mol Psychiatry 8:916-924. CrossRef Medline

Bayés A, van de Lagemaat LN, Collins MO, Croning MD, Whittle IR, Choudhary JS, Grant SG (2011) Characterization of the proteome, diseases and evolution of the human postsynaptic density. Nat Neurosci 14:19-21. CrossRef Medline

Bernardinelli Y, Nikonenko I, Muller D (2014) Structural plasticity: mechanisms and contribution to developmental psychiatric disorders. Front Neuroanat 8:123. CrossRef Medline

Bonhoeffer T, Yuste R (2002) Spine motility. Phenomenology, mechanisms, and function. Neuron 35:1019-1027. CrossRef Medline

Caroni P, Donato F, Muller D (2012) Structural plasticity upon learning: regulation and functions. Nat Rev Neurosci 13:478-490. CrossRef Medline

Chen J, Yu S, Fu Y, Li X (2014) Synaptic proteins and receptors defects in autism spectrum disorders. Front Cell Neurosci 8:276. CrossRef Medline

Chen X, Wang X, Hossain S, O’Neill FA, Walsh D, Pless L, Chowdari KV, Nimgaonkar VL, Schwab SG, Wildenauer DB, Sullivan PF, van den Oord E, Kendler KS (2006) Haplotypes spanning SPEC2, PDZ-GEF2 and ACSL6 genes are associated with schizophrenia. Hum Mol Genet 15: 3329-3342. CrossRef Medline

Cheng R, Juo SH, Loth JE, Nee J, Iossifov I, Blumenthal R, Sharpe L, Kanyas K, Lerer B, Lilliston B, Smith M, Trautman K, Gilliam TC, Endicott J, Baron M (2006) Genome-wide linkage scan in a large bipolar disorder sample from the National Institute of Mental Health genetics initiative suggests putative loci for bipolar disorder, psychosis, suicide, and panic disorder. Mol Psychiatry 11:252-260. CrossRef Medline

De Rubeis S, He X, Goldberg AP, Poultney CS, Samocha K, Cicek AE, Kou Y, Liu L, Fromer M, Walker S, Singh T, Klei L, Kosmicki J, Shih-Chen F, Aleksic B, Biscaldi M, Bolton PF, Brownfeld JM, Cai J, Campbell NG, et al. (2014) Synaptic, transcriptional and chromatin genes disrupted in autism. Nature 515:209-215. CrossRef Medline

de Rooij J, Boenink NM, van Triest M, Cool RH, Wittinghofer A, Bos JL (1999) PDZ-GEF1, a guanine nucleotide exchange factor specific for Rap1 and Rap2. J Biol Chem 274:38125-38130. CrossRef Medline

Dumitriu D, Hao J, Hara Y, Kaufmann J, Janssen WG, Lou W, Rapp PR, Morrison JH (2010) Selective changes in thin density and morphology in monkey prefrontal cortex correlate with aging-related cognitive impairment. J Neurosci 30:7507-7515. CrossRef Medline

Easley-Neal C, Fierro J Jr, Buchanan J, Washbourne P (2013) Late recruit- ment of synapsin to nascent synapses is regulated by Cdk5. Cell Rep 3:1199-1212. CrossRef Medline

Etkin A, Egner T, Kalisch R (2011) Emotional processing in anterior cingulate and medial prefrontal cortex. Trends Cogn Sci 15: 85-93.1. Medline

Euston DR, Gruber AJ, McNaughton BL (2012) The role of medial prefrontal cortex in memory and decision making. Neuron 76:1057-1070. CrossRef Medline

Fu M, Zuo Y (2011) Experience-dependent structural plasticity in the cortex. Trends Neurosci 34:177-187. CrossRef Medline

Fu Z, Lee SH, Simonetta A, Hansen J, Sheng M, Pak DT (2007) Differential roles of Rap1 and Rap2 small GTPases in neurite retraction and synapse elimination in hippocampal spiny neurons. J Neurochem 100:118-131. CrossRef Medline

Fukumoto K, Morita T, Mayanagi T, Tanokashira D, Yoshida T, Sakai A, Sobue K (2009) Detrimental effects of glucocorticoids on neuronal migration during brain development. Mol Psychiatry 14:1119-1131. CrossRef Medline

Holmes A, Wellman CL (2009) Stress-induced prefrontal reorganization and executive dysfunction in rodents. Neurosci Biobehav Rev 33: 773-783. CrossRef Medline

Hutsler JJ, Zhang H (2010) Increased dendritic spine densities on cortical projection neurons in autism spectrum disorders. Brain Res 14:1309: 83-13094. CrossRef

Irwin SA, Galvez R, Greenough WT (2000) Dendritic spine structural anomalies in fragile-X mental retardation syndrome. Cereb Cortex 10: 1038-1044. CrossRef Medline

Isaac JT, Nicoll RA, Malenka RC (1995) Evidence for silent synapses: implications for the expression of LTP. Neuron 15:427-434. CrossRef Medline

Ishii H, Baffa R, Numata SI, Murakumo Y, Rattan S, Inoue H, Mori M, Fidanza V, Alder H, Croce CM (1999) The FEZ1 gene at chromosome 8 p22 encodes a leucine-zipper protein, and its expression is altered in multiple human tumors. Proc Natl Acad Sci U S A 96:3928-3933. CrossRef Medline

Jiang M, Chen G (2006) High Ca2+-phosphate transfection efficiency in low-density neuronal cultures. Nat Protoc 1:695-700. CrossRef Medline

Kendler KS, MacLean CJ, Ma Y, O’Neill FA, Walsh D, Straub RE (1999) Marker-to-marker linkage disequilibrium on chromosomes 5q, 6p, and $8 p$ in Irish high-density schizophrenia pedigrees. Am J Med Genet 88:29 33. CrossRef Medline

Kinou M, Takizawa R, Marumo K, Kawasaki S, Kawakubo Y, Fukuda M, Kasai K (2013) Differential spatiotemporal characteristics of the prefrontal hemodynamic response and their association with functional impairment in schizophrenia and major depression. Schizophr Res 150: 459-467. CrossRef Medline

Konno D, Ko JA, Usui S, Hori K, Maruoka H, Inui M, Fujikado T, Tano Y, Suzuki T, Tohyama K, Sobue K (2002) The postsynaptic density and dendritic raft localization of PSD-Zip70, which contains an $N$-myristoylation sequence and leucinezipper motifs. J Cell Sci 115:4695-4706. CrossRef Medline

Lee KJ, Lee Y, Rozeboom A, Lee JY, Udagawa N, Hoe HS, Pak DT (2011) Requirement for Plk2 in orchestrated ras and rap signaling, homeostatic structural plasticity, and memory. Neuron 69:957-973. CrossRef Medline

Levy R, Goldman-Rakic PS (2000) Segregation of working memory functions within the dorsolateral prefrontal cortex. Exp Brain Res 133:23-32. CrossRef Medline

$\mathrm{Lu}$ W, Roche KW (2012) Posttranslational regulation of AMPA receptor trafficking and function. Curr Opin Neurobiol 22:470-479. CrossRef Medline

Maruoka H, Konno D, Hori K, Sobue K (2005) Collaboration of PSD-Zip70 with its binding partner, SPAR, in dendritic spine maturity. J Neurosci 25:1421-1430. CrossRef Medline

Matsuzaki M, Ellis-Davies GC, Nemoto T, Miyashita Y, Iino M, Kasai H (2001) Dendritic spine geometry is critical for AMPA receptor expression in hippocampal CAl pyramidal neurons. Nat Neurosci 4:10861092. CrossRef Medline

Miller EK, Erickson CA, Desimone R (1996) Neural mechanisms of visual working memory in prefrontal cortex of the macaque. J Neurosci 16 : 5154-5167. Medline

Mozhayeva MG, Sara Y, Liu X, Kavalali ET (2002) Development of vesicle pools during maturation of hippocampal synapses. J Neurosci 22: 654-665. Medline

Nimchinsky EA, Sabatini BL, Svoboda K (2002) Structure and function of dendritic spines. Annu Rev Physiol 64:313-353. Medline 
Nishiyama J, Yasuda R (2015) Biochemical computation for spine structural plasticity. Neuron 87:63-75. CrossRef Medline

Niwa M, Kamiya A, Murai R, Kubo K, Gruber AJ, Tomita K, Lu L, Tomisato S, Jaaro-Peled H, Seshadri S, Hiyama H, Huang B, Kohda K, Noda Y, O’Donnell P, Nakajima K, Sawa A, Nabeshima T (2010) Knockdown of DISC1 by in utero gene transfer disturbs postnatal dopaminergic maturation in the frontal cortex and leads to adult behavioral deficits. Neuron 65:480-489. CrossRef Medline

Okabe T, Sobue K (1987) Identification of a new $84 / 82 \mathrm{kDa}$ calmodulinbinding protein, which also interacts with actin filaments, tubulin and spectrin, as synapsin I. FEBS Lett 213:184-188. CrossRef Medline

Pak DT, Yang S, Rudolph-Correia S, Kim E, Sheng M (2001) Regulation of dendritic spine morphology by SPAR, a PSD-95-associated RapGAP. Neuron 31:289-303. CrossRef Medline

Papanikolaou K, Paliokosta E, Gyftodimou J, Kolaitis G, Vgenopoulou S, Sarri C, Tsiantis J (2006) A case of partial trisomy of chromosome 8p associated with autism. J Autism Dev Disord 36:705-709. CrossRef Medline

Park N, Juo SH, Cheng R, Liu J, Loth JE, Lilliston B, Nee J, Grunn A, Kanyas K, Lerer B, Endicott J, Gilliam TC, Baron M (2004) Linkage analysis of psychosis in bipolar pedigrees suggests novel putative loci for bipolar disorder and shared susceptibility with schizophrenia. Mol Psychiatry 9:1091-1099. CrossRef Medline

Penzes P, Cahill ME, Jones KA, VanLeeuwen JE, Woolfrey KM (2011) Dendritic spine pathology in neuropsychiatric disorders. Nat Neurosci 14: 285-293. CrossRef Medline

Ragland JD, Yoon J, Minzenberg MJ, Carter CS (2007) Neuroimaging of cognitive disability in schizophrenia: search for a pathophysiological mechanism. Int Rev Psychiatry 19:417-427. CrossRef Medline

Renger JJ, Egles C, Liu G (2001) Developmental switch in neurotransmitter flux enhances synaptic efficacy by affecting AMPA receptor activation. Neuron 29:469-484. CrossRef Medline

Rumpel S, Hatt H, Gottmann K (1998) Silent synapses in the developing rat visual cortex: evidence for postsynaptic expression of synaptic plasticity. J Neurosci 18:8863-8874. Medline

Ryu J, Futai K, Feliu M, Weinberg R, Sheng M (2008) Constitutively active Rap2 transgenic mice display fewer dendritic spines, reduced extracellular signal-regulated kinase signaling, enhanced long-term depression, and impaired spatial learning and fear extinction. J Neurosci 28:8178-8188. CrossRef Medline

Sala C, Segal M (2014) Dendritic spines: the locus of structural and functional plasticity. Physiol Rev 94:141-188. CrossRef Medline

Shyn SI, Shi J, Kraft JB, Potash JB, Knowles JA, Weissman MM, Garriock HA, Yokoyama JS, McGrath PJ, Peters EJ, Scheftner WA, Coryell W, Lawson WB, Jancic D, Gejman PV, Sanders AR, Holmans P, Slager SL, Levinson DF, Hamilton SP (2011) Novel loci for major depression identified by genome-wide association study of Sequenced Treatment Alternatives to Relieve Depression and meta-analysis of three studies. Mol Psychiatry 16:202-215. CrossRef Medline

Tabarés-Seisdedos R, Rubenstein JL (2009) Chromosome 8p as a potential hub for developmental neuropsychiatric disorders: implications for schizophrenia, autism and cancer. Mol Psychiatry 14:563-589. CrossRef Medline
Tachibana T, Imanaka T, Ko JA, Tadokoro S, Nishida W, Sobue K (1999) Isolation of PSD-Zip70, a novel protein containing leucine zipper motif. Cell Struct Funct 24:582.

Tomioka NH, Yasuda H, Miyamoto H, Hatayama M, Morimura N, Matsumoto Y, Suzuki T, Odagawa M, Odaka YS, Iwayama Y, Won Um J, Ko J, Inoue Y, Kaneko S, Hirose S, Yamada K, Yoshikawa T, Yamakawa K, Aruga J (2014) Elfn1 recruits presynaptic mGluR7 in trans and its loss results in seizures. Nat Commun 5:4501. CrossRef Medline

Uechi Y, Bayarjargal M, Umikawa M, Oshiro M, Takei K, Yamashiro Y, Asato T, Endo S, Misaki R, Taguchi T, Kariya K (2009) Rap2 function requires palmitoylation and recycling endosome localization. Biochem Biophys Res Commun 378:732-737. CrossRef Medline

Usui S, Konno D, Hori K, Maruoka H, Okabe S, Fujikado T, Tano Y, Sobue K (2003) Synaptic targeting of PSD-Zip45 (Homer 1c) and its involvement in the synaptic accumulation of F-actin. J Biol Chem 278:10619-10628. CrossRef Medline

Vecchione A, Ishii H, Shiao YH, Trapasso F, Rugge M, Tamburrino JF, Murakumo Y, Alder H, Croce CM, Baffa R (2001) Altered expression of Fezl protein in gastric carcinoma. Clin Cancer Res 7:1546-1552. Medline

Vecchione A, Baldassarre G, Ishii H, Nicoloso MS, Belletti B, Petrocca F, Zanesi N, Fong LY, Battista S, Guarnieri D, Baffa R, Alder H, Farber JL, Donovan PJ, Croce CM (2007) Fez1/Lzts1 absence impairs Cdk1/ $\mathrm{Cdc} 25 \mathrm{C}$ interaction during mitosis and predisposes mice to cancer development. Cancer Cell 11:275-289. CrossRef Medline

Wang JQ, Arora A, Yang L, Parelkar NK, Zhang G, Liu X, Choe ES, Mao L (2005) Phosphorylation of AMPA receptors: mechanisms and synaptic plasticity. Mol Neurobiol 32:237-249. CrossRef Medline

Wild-Wall N, Falkenstein M, Gajewski PD (2011) Age-related differences in working memory performance in a 2-back task. Front Psychol 2:186-198. CrossRef Medline

Yasuda H, Mukai H (2015) Turning off of GluN2B subunits and on of CICR in hippocampal LTD induction after developmental GluN2 subunit switch. Hippocampus. Advance online publication. CrossRef Medline

Zang YF, Jin Z, Weng XC, Zhang L, Zeng YW, Yang L, Wang YF, Seidman LJ, Faraone SV (2005) Functional MRI in attention-deficit hyperactivity disorder: evidence for hypofrontality. Brain Dev 27:544-550. CrossRef Medline

Zhao S, Ting JT, Atallah HE, Qiu L, Tan J, Gloss B, Augustine GJ, Deisseroth K, Luo M, Graybiel AM, Feng G (2011) Cell type-specific channelrhodopsin-2 transgenic mice for optogenetic dissection of neural circuitry function. Nat Methods 8:745-752. CrossRef Medline

Zhu JJ, Qin Y, Zhao M, Van Aelst L, Malinow R (2002) Ras and Rap control AMPA receptor trafficking during synaptic plasticity. Cell 110:443-455. CrossRef Medline

Zhu Y, Pak D, Qin Y, McCormack SG, Kim MJ, Baumgart JP, Velamoor V, Auberson YP, Osten P, van Aelst L, Sheng M, Zhu JJ (2005) Rap2-JNK removes synaptic AMPA receptors during depotentiation. Neuron 46: 905-916. CrossRef Medline

Zubenko GS, Maher BS, Hughes HB 3rd, Zubenko WN, Scott Stiffler J, Marazita ML (2004) Genome-wide linkage survey for genetic loci that affect the risk of suicide attempts in families with recurrent, early-onset, major depression. Am J Med Genet B Neuropsychiatr Genet 129B:47-54. CrossRef Medline 